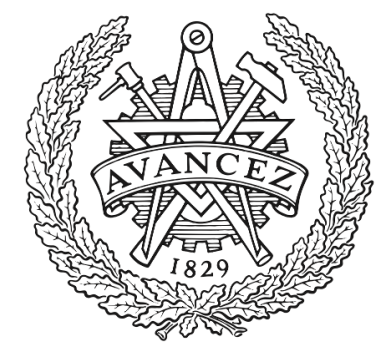

CHALMERS

UNIVERSITY OF TECHNOLOGY

\title{
Inexact Newton-Type Optimization with Iterated Sensitivities
}

Downloaded from: https://research.chalmers.se, 2023-04-26 08:30 UTC

Citation for the original published paper (version of record):

Quirynen, R., Gros, S., Diehl, M. (2018). Inexact Newton-Type Optimization with Iterated

Sensitivities. SIAM Journal on Optimization, 28(1): 74-95. http://dx.doi.org/10.1137/16M1079002

N.B. When citing this work, cite the original published paper. 


\title{
INEXACT NEWTON-TYPE OPTIMIZATION WITH ITERATED SENSITIVITIES*
}

\author{
RIEN QUIRYNEN ${ }^{\dagger}$, SÉBASTIEN GROS ${ }^{\ddagger}$, AND MORITZ DIEHL ${ }^{\S}$
}

\begin{abstract}
This paper presents and analyzes an inexact Newton-type optimization method based on iterated sensitivities (INIS). A particular class of nonlinear programming (NLP) problems is considered, where a subset of the variables is defined by nonlinear equality constraints. The proposed algorithm considers any problem-specific approximation for the Jacobian of these constraints. Unlike other inexact Newton methods, the INIS-type optimization algorithm is shown to preserve the local convergence properties and the asymptotic contraction rate of the Newton-type scheme for the feasibility problem yielded by the same Jacobian approximation. The INIS approach results in a computational cost which can be made close to that of the standard inexact Newton implementation. In addition, an adjoint-free (AF-INIS) variant of the approach is presented which, under certain conditions, becomes considerably easier to implement than the adjoint based scheme. The applicability of these results is motivated specifically for dynamic optimization problems. In addition, the numerical performance of a corresponding open-source implementation is illustrated.
\end{abstract}

Key words. Newton-type methods, optimization algorithms, direct optimal control, collocation methods

AMS subject classifications. 49M15, 90C30, 65M70

DOI. $10.1137 / 16 \mathrm{M} 1079002$

1. Introduction. The present paper considers Newton-type optimization algorithms [19] for a class of nonlinear programming (NLP) problems

$$
\begin{array}{ll}
\min _{z, w} & f(z, w) \\
\text { s.t. } & g(z, w)=0, \\
& h(z, w)=0,
\end{array}
$$

where $z \in \mathbb{R}^{n_{z}}$ and $w \in \mathbb{R}^{n_{\mathrm{w}}}$ are the optimization variables. The objective and constraint functions are defined as $f: \mathbb{R}^{n_{\mathrm{z}}} \times \mathbb{R}^{n_{\mathrm{w}}} \rightarrow \mathbb{R}, g: \mathbb{R}^{n_{\mathrm{z}}} \times \mathbb{R}^{n_{\mathrm{w}}} \rightarrow \mathbb{R}^{n_{g}}$ and $h: \mathbb{R}^{n_{\mathrm{z}}} \times \mathbb{R}^{n_{\mathrm{w}}} \rightarrow \mathbb{R}^{n_{\mathrm{h}}}$, respectively, and they are assumed to be twice continuously differentiable in all arguments. The subset of the variables $z$ and the constraint function $g(\cdot)$ is selected such that $n_{\mathrm{g}}=n_{\mathrm{z}}$ and such that the Jacobian $\frac{\partial g(z, w)}{\partial z} \in \mathbb{R}^{n_{\mathrm{z}} \times n_{\mathrm{z}}}$ is invertible. It follows that the variables $z$ are implicitly defined as functions of $w$ via the nonlinear equality constraints $g(z, w)=0$. Throughout the paper, this set

\footnotetext{
*Received by the editors June 9, 2016; accepted for publication (in revised form) July 7, 2017; published electronically January 9, 2018.

http://www.siam.org/journals/siopt/28-1/M107900.html

Funding: This research was supported by the EU via ERC-HIGHWIND (259 166), FP7-ITNTEMPO (607 957), H2020-ITN-AWESCO (642 682), and by the DFG in context of the Research Unit FOR 2401. At the time of initial submission, the first author held a Ph.D. fellowship of the Research Foundation - Flanders (FWO), and he is currently affiliated with the Mitsubishi Electric Research Laboratories (MERL) in Cambridge, Massachusetts.

${ }^{\dagger}$ Department ESAT-STADIUS, KU Leuven University, 3001 Leuven, Belgium (rien.quirynen@ gmail.com).

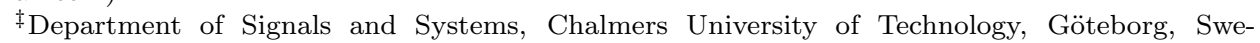
den (grosse@chalmers.se), and Freiburg Institute for Advanced Studies (FRIAS), 79104 Freiburg, Germany.

$\S$ Department of Microsystems Engineering - IMTEK, University of Freiburg, 79110 Freiburg, Germany (moritz.diehl@imtek.uni-freiburg.de).
} 
of constraints in Eq. (1b) will be referred to as the forward problem, which delivers $z^{\star}(\bar{w})$ by solving the corresponding system

$$
g(z, \bar{w})=0 \quad \text { for a given value } \bar{w} .
$$

Some interesting examples of such problem formulations result from a simultaneous approach to dynamic optimization $[2,10]$, where the forward problem imposes the system dynamics and therefore typically corresponds to a numerical simulation of differential equations. A popular example of such an approach is direct collocation [5], where the forward problem consists of the collocation equations, possibly including also the continuity conditions.

We are interested in solving the forward problem in (2) using Newton-type schemes that do not rely on an exact factorization of $g_{z}:=\frac{\partial g}{\partial z}$, but use instead a full-rank approximation $M \approx g_{z}$. This Jacobian approximation can be used directly in a Newton-type method to solve the forward problem by steps

$$
\Delta z=-M^{-1} g(\bar{z}, \bar{w})
$$

where $\bar{z}$ denotes the current guess and the full-step update in each Newton-type iteration can be written as $\bar{z}^{+}=\bar{z}+\Delta z$. Even though local convergence properties for Newton-type optimization have been studied extensively in $[6,16,19,36]$, this paper presents a novel contribution regarding the connection between the accuracy of the Jacobian approximation $M$ and the local contraction rate of the corresponding optimization algorithm. A Newton-type method with inexact derivatives does not converge to a solution of the original nonlinear optimization problem, unless adjoint derivatives are evaluated in order to compute the correct gradient of the Lagrangian $[9$, 22]. It has been pointed out by [39] that the locally linear convergence rate of the resulting inexact Newton (IN) based optimization scheme is not strongly connected to the contraction of the iterations for the inner forward problem in (3). More specifically, it is possible that the Jacobian approximation $M$ results in a fast contraction of the forward problem alone, while the optimization algorithm based on the same Jacobian approximation diverges. In contrast, the proposed INIS algorithm will be shown to have the same asymptotic contraction rate.

In this work, for the sake of simplicity, we omit (possibly nonlinear) inequality constraints in the NLP (1). Note however that our discussion on the local convergence of Newton-type optimization methods can be readily extended to the general case of inequality-constrained optimization. Such an extension can be based on techniques from sequential quadratic programming (SQP) where, under mild conditions, the active set can be shown to be locally stable for the subproblems $[9,11,38]$. Hence, for the purpose of a local convergence analysis, the equality constraints in (1c) could additionally comprise the locally active inequality constraints. This observation is illustrated further in the numerical case study of this paper. Alternatively, the extension to inequality-constrained optimization can similarly be carried out in the context of interior point methods [5, 37]. Convergence results for Newton-type optimization based on inexact derivative information can be found in [30,34] for SQP or in $[4,48]$ for nonlinear interior point (IP) methods. An alternative approach makes use of inexact solutions to the linearized subproblems in order to reduce the overall computational burden of the Newton-type scheme as discussed in [14, 15, 30,35]. Note that other variants of inexact Newton-type algorithms exist, e.g., allowing locally superlinear convergence $[22,29]$ based on quasi-Newton Jacobian updates. In the case of optimal control for differential-algebraic equations, even quadratic convergence rates [31] have been observed under certain conditions. 
1.1. Contributions and outline. The main contribution of the present paper is the inexact Newton method with iterated sensitivities (INIS) that allows one to recover a strong connection between the local contraction rate of the forward problem and the local convergence properties of the resulting Newton-type optimization algorithm. More specifically, local contraction based on the Jacobian approximation for the forward problem is necessary and, under mild conditions, even sufficient for local convergence of the INIS-type optimization scheme. The article presents an efficient implementation of the INIS algorithm, resulting in a computational cost close to that of the standard inexact Newton implementation. Note that this Newton-type scheme shows a particular resemblance to the lifted Newton method in [1], based on a lifting of the forward sensitivities. This connection is also discussed in [44], in the context of collocation methods for direct optimal control.

In addition, an adjoint-free (AF-INIS) variant for Newton-type optimization is proposed. This alternative approach can be interesting whenever the algorithm can be carried out independently of the respective values for the multipliers corresponding to the equality constraints, but it generally does not preserve the local convergence properties of the forward scheme. As discussed further, an adjoint-free implementation can, however, be attractive in case of a sequence of nontrivial operations, e.g., resulting from a numerical simulation in dynamic optimization. An open-source implementation of these novel INIS-type techniques for simultaneous direct optimal control is proposed as part of the ACADO toolkit. Throughout the article, theoretical results are illustrated using toy examples of quadratic and nonlinear programming problems. In addition, the numerical performance of the open-source implementation is shown on the benchmark case study of the optimal control for a chain of masses.

The paper is organized as follows. Section 2 briefly presents standard Newtontype optimization methods. Section 3 then proposes and analyzes the inexact Newton method based on iterated sensitivities (INIS) as an alternative implementation of inexact Newton-type optimization. An adjoint-free variant of the INIS-type optimization algorithm is presented in section 4. An important application of the proposed schemes for simultaneous approaches of direct optimal control is presented in section 5, including numerical results based on a specific open-source implementation. Section 6 finally concludes this paper.

2. Newton-type optimization. The Lagrange function for the NLP (1) reads as $\mathcal{L}(y, \lambda)=f(y)+\mu^{\top} g(y)+\nu^{\top} h(y)$, where $y:=\left[\begin{array}{ll}z^{\top} & w^{\top}\end{array}\right]^{\top} \in \mathbb{R}^{n_{y}}$ denotes all primal variables. In addition, $c(y):=\left[\begin{array}{ll}g(y)^{\top} & h(y)^{\top}\end{array}\right]^{\top}, \lambda:=\left[\begin{array}{ll}\mu^{\top} & \nu^{\top}\end{array}\right]^{\top} \in \mathbb{R}^{n_{c}}$ is defined, and $n_{\mathrm{c}}=n_{\mathrm{g}}+n_{\mathrm{h}}$, where $\mu \in \mathbb{R}^{n_{\mathrm{g}}}, \nu \in \mathbb{R}^{n_{\mathrm{h}}}$, respectively, denote the multipliers for the nonlinear equality constraints in (1b) and (1c). The first-order necessary conditions for optimality are then defined as

$$
\begin{aligned}
\nabla_{y} \mathcal{L}(y, \lambda): & \nabla_{y} f(y)+\sum_{i=1}^{n_{\mathrm{c}}} \lambda_{i} \nabla_{y} c_{i}(y) & =0, \\
\nabla_{\lambda} \mathcal{L}(y, \lambda): & c(y) & =0
\end{aligned}
$$

and are generally referred to as the Karush-Kuhn-Tucker (KKT) conditions [37]. Note that a more compact notation is used to denote the gradient of a scalar function, i.e., this is the transpose of the Jacobian $\nabla_{y} \mathcal{L}(\cdot)=\frac{\partial \mathcal{L}}{\partial y}(\cdot)^{\top}=\mathcal{L}_{y}(\cdot)^{\top}$. We further generalize this operator as $\nabla_{y} c(\cdot)=\left[\begin{array}{lll}\nabla_{y} c_{1}(\cdot) & \cdots & \nabla_{y} c_{n_{c}}(\cdot)\end{array}\right]=\frac{\partial c}{\partial y}(\cdot)^{\top}=c_{y}(\cdot)^{\top}$. This nonlinear system of equations can also be written in the compact notation 


$$
\mathcal{F}(y, \lambda)=\left[\begin{array}{c}
\nabla_{y} \mathcal{L}(y, \lambda) \\
c(y)
\end{array}\right]=0
$$

Each local minimizer $\left(y^{\star}, \lambda^{\star}\right)$ of the NLP (1) is assumed to be a regular KKT point $\mathcal{F}\left(y^{\star}, \lambda^{\star}\right)=0$ as defined next. For this purpose, we rely on the linear independence constraint qualification (LICQ) and the second-order sufficient conditions (SOSC) for optimality, of which the latter requires that the Hessian of the Lagrangian is strictly positive definite in the directions of the critical cone [37].

DEFINITION 2.1. A minimizer of an equality constrained NLP is called a regular $K K T$ point, if both LICQ and SOSC are satisfied at this KKT point.

2.1. Newton-type methods. Newton-type optimization proceeds with applying a variant of Newton's method $[17,19]$ to find a solution to the KKT system in (5). Note that an exact Newton iteration on the KKT conditions reads as

$$
\underbrace{\left[\begin{array}{cc}
\nabla_{y}^{2} \mathcal{L}(\bar{y}, \bar{\lambda}) & c_{y}^{\top}(\bar{y}) \\
c_{y}(\bar{y}) & \mathbb{0}
\end{array}\right]}_{=J(\bar{y}, \bar{\lambda})}\left[\begin{array}{c}
\Delta y \\
\Delta \lambda
\end{array}\right]=-\underbrace{\left[\begin{array}{c}
\nabla_{y} \mathcal{L}(\bar{y}, \bar{\lambda}) \\
c(\bar{y})
\end{array}\right]}_{=\mathcal{F}(\bar{y}, \bar{\lambda})},
$$

where $c_{y}(\bar{y}):=\frac{\partial c}{\partial y}(\bar{y})$ denotes the Jacobian matrix and the values $\bar{y}$ and $\bar{\lambda}$ denote the primal and dual variables at the current guess. In the following, we will refer to the exact Newton iteration using the compact notation

$$
J(\bar{y}, \bar{\lambda})\left[\begin{array}{l}
\Delta y \\
\Delta \lambda
\end{array}\right]=-\mathcal{F}(\bar{y}, \bar{\lambda})
$$

where the exact Jacobian matrix is defined as $J(\bar{y}, \bar{\lambda}):=\frac{\partial \mathcal{F}}{\partial(y, \lambda)}(\bar{y}, \bar{\lambda})$. In this work, a full-step update of the primal and dual variables is considered for simplicity in each iteration, i.e., $\bar{y}^{+}=\bar{y}+\Delta y$ and $\bar{\lambda}^{+}=\bar{\lambda}+\Delta \lambda$, even though globalization strategies are typically used to guarantee convergence $[5,37]$.

As mentioned earlier, many Newton-type optimization methods have been proposed that result in desirable local convergence properties at a considerably reduced computational cost by either forming an approximation of the KKT matrix $J(\cdot)$ or by solving the linear system (6) approximately [35]. For example, the family of quasi-Newton methods $[18,37]$ is based on the approximation of the Hessian of the Lagrangian $\tilde{H} \approx H:=\nabla_{y}^{2} \mathcal{L}$ using only first-order derivative information. Other Newton-type optimization algorithms even use an inexact Jacobian for the nonlinear constraints $[9,22,31,47]$ as discussed next.

2.2. Adjoint-based inexact Newton (IN). Let us consider the invertible Jacobian approximation $M \approx g_{z}$ in the Newton-type method of (3) to solve the forward problem. The resulting inexact Newton method, aimed at solving the KKT conditions for the NLP in (5), iteratively solves the following linear system:

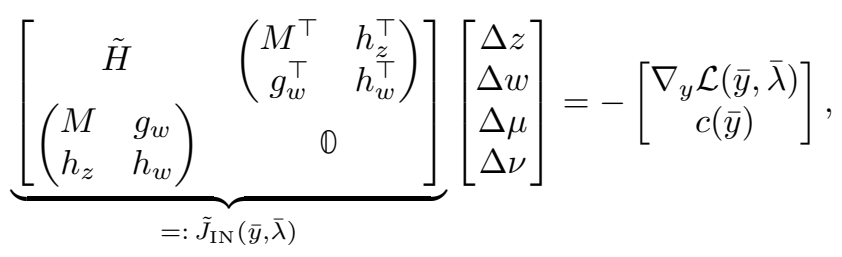

where the right-hand side of the system is exact as in (6) and an approximation of the Hessian $\tilde{H} \approx \nabla_{y}^{2} \mathcal{L}(\bar{y}, \bar{\lambda})$ has been introduced for the sake of completeness. 
Note that the gradient of the Lagrangian $\nabla_{y} \mathcal{L}(\cdot)$ can be evaluated efficiently using adjoint differentiation techniques, such that the scheme is often referred to as an adjoint-based inexact Newton (IN) method [9, 22]. The corresponding convergence analysis will be discussed later. Algorithm 1 describes an implementation to solve the adjoint-based IN system in (8). It relies on a numerical elimination of the variables $\Delta z=-M^{-1}\left(g(\bar{y})+g_{w} \Delta w\right)$ and $\Delta \mu$ such that a smaller system is solved in the variables $\Delta w, \Delta \nu$, which can be expanded back into the full variable space. Note that one recovers the Newton-type iteration on the forward problem $\Delta z=-M^{-1} g(\bar{y})$ for a fixed value $\bar{w}$, i.e., in case $\Delta w=0$.

Remark 2.2. The matrix $\tilde{Z}^{\top}:=\left[-g_{w}^{\top} M^{-\top}, \mathbb{1}_{n_{\mathrm{w}}}\right]$ in step 1 of Algorithm 1 is an approximation for $Z^{\top}:=\left[-g_{w}^{\top} g_{z}^{-\top}, \mathbb{1}_{n_{\mathrm{w}}}\right]$, which denotes a basis for the null space of the constraint Jacobian $g_{y} Z=\mathbb{O}$ such that $Z^{\top} \nabla_{y} \mathcal{L}(\bar{y}, \bar{\lambda})=Z^{\top}\left(\nabla_{y} f(\bar{y})+\nabla_{y} h(\bar{y}) \bar{\nu}\right)$. When using instead the approximate matrix $\tilde{Z}$, this results in the following correction of the gradient term:

$$
\tilde{Z}^{\top} \nabla_{y} \mathcal{L}(\bar{y}, \bar{\lambda})=\tilde{Z}^{\top}\left(\nabla_{y} f(\bar{y})+\nabla_{y} h(\bar{y}) \bar{\nu}\right)-\left(\left(g_{z} M^{-1}-\mathbb{1}_{n_{z}}\right) g_{w}\right)^{\top} \bar{\mu} .
$$

In addition, let us write the first $n_{\mathrm{z}}$ expressions in (8) as follows:

$$
M^{\top} \Delta \mu=-\left[\mathbb{1}_{n_{z}} \quad 0\right]\left(\nabla_{y} \mathcal{L}(\bar{y}, \bar{\lambda})+\tilde{H} \Delta y+h_{y}^{\top} \Delta \nu\right),
$$

which has been used to obtain the expansion step for $\Delta \mu$ in Algorithm 1.

2.3. Newton-type local convergence. One iteration of the adjoint-based IN method solves the linear system in (8), which can be written in the following compact form:

$$
\tilde{J}_{\mathrm{IN}}(\bar{y}, \bar{\lambda})\left[\begin{array}{l}
\Delta y \\
\Delta \lambda
\end{array}\right]=-\mathcal{F}(\bar{y}, \bar{\lambda})
$$

where $\mathcal{F}(\cdot)$ denotes the exact KKT right-hand side in (6). The convergence of this scheme then follows the classical and well-known local contraction theorem from $[6$, $19,22,39]$. We use a particular version of this theorem from [20], providing sufficient and necessary conditions for the existence of a neighborhood of the solution where the Newton-type iteration converges. Let $\rho(P)$ denote the spectral radius, i.e., the maximum absolute value of the eigenvalues for the square matrix $P$.

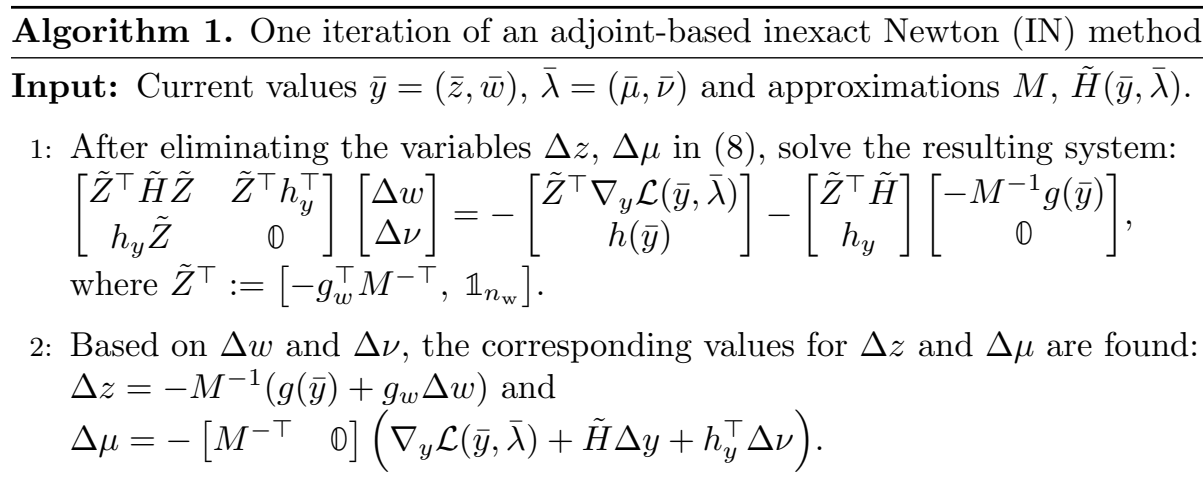

Output: New values $\bar{y}^{+}=\bar{y}+\Delta y$ and $\bar{\lambda}^{+}=\bar{\lambda}+\Delta \lambda$. 
TheOREM 2.3 (Local Newton-type contraction [20]). We consider the twice continuously differentiable function $\mathcal{F}(y, \lambda)$ from $(5)$ and the regular $K K T$ point $\mathcal{F}\left(y^{\star}, \lambda^{\star}\right)$ $=0$ from Definition 2.1. We then apply the Newton-type iteration in (11), where $\tilde{J}_{\mathrm{IN}}(\bar{y}, \bar{\lambda}) \approx J(\bar{y}, \bar{\lambda})$ is additionally assumed to be continuously differentiable and invertible in a neighborhood of the solution. If all eigenvalues of the iteration matrix have a modulus smaller than 1 , i.e., if the spectral radius satisfies

$$
\kappa^{\star}:=\rho\left(\tilde{J}_{\mathrm{IN}}\left(y^{\star}, \lambda^{\star}\right)^{-1} J\left(y^{\star}, \lambda^{\star}\right)-\mathbb{1}_{n_{\mathcal{F}}}\right)<1,
$$

then this fixed point $\left(y^{\star}, \lambda^{\star}\right)$ is asymptotically stable, where $n_{\mathcal{F}}=n_{\mathrm{y}}+n_{\mathrm{g}}+n_{\mathrm{h}}$. Additionally, the iterates $(\bar{y}, \bar{\lambda})$ converge linearly to the KKT point $\left(y^{\star}, \lambda^{\star}\right)$ with the asymptotic contraction rate $\kappa^{\star}$ when initialized sufficiently close. On the other hand, if $\kappa^{\star}>1$, then the fixed point $\left(y^{\star}, \lambda^{\star}\right)$ is unstable.

A proof for Theorem 2.3 can be found in [20,41], based on a classical stability result from nonlinear systems theory.

Remark 2.4. The inexact Newton method relies on the standard assumption that the Jacobian and Hessian approximations $M$ and $\tilde{H}$ in (8) are such that the corresponding matrix $\tilde{J}_{\mathrm{IN}}(\bar{y}, \bar{\lambda})$ is invertible. However, in addition, Theorem 2.3 requires that $\tilde{J}_{\mathrm{IN}}(\cdot)$ is continuously differentiable in a neighborhood of the solution. This assumption is satisfied, e.g., for an exact Newton method, for fixed Jacobian approximations, as well as for the generalized Gauss-Newton (GGN) method for least squares type optimization $[8,20]$. This theorem on local Newton-type convergence will therefore be sufficient for our discussion, even though more advanced results exist [16, 19].

Remark 2.5. As mentioned earlier in the introduction, an inequality constrained problem can be solved with any of the proposed Newton-type optimization algorithms, in combination with techniques from either SQP or interior point methods to treat the inequality constraints. Let us consider a local minimizer which is assumed to be regular, i.e., it satisfies the linear independence constraint qualification (LICQ), the strict complementarity condition, and the second-order sufficient conditions (SOSC) as defined in [37]. In this case, the primal-dual central path associated with this minimizer is locally unique when using an interior point method. In case of an SQP method, under mild conditions on the Hessian and Jacobian approximations, the corresponding active set is locally stable in a neighborhood of the minimizer, i.e., the solution of each QP subproblem has the same active set as the original NLP [9, 11, 46]. Hence, for the purpose of a local convergence analysis, the equality constraints in (1c) could additionally comprise the active inequality constraints in a neighborhood of the local minimizer.

2.4. A motivating QP example. In this paper, we are interested in the existence of a connection between the Newton-type iteration on the forward problem (3) being locally contractive, i.e., $\kappa_{\mathrm{F}}^{\star}:=\rho\left(M^{-1} g_{z}\left(z^{\star}, \bar{w}\right)-\mathbb{1}_{n_{\mathrm{z}}}\right)<1$, and the local convergence for the corresponding Newton-type optimization algorithm as defined by Theorem 2.3. From the detailed discussion in [7, 39, 40], we know that contraction for the forward problem is neither sufficient nor necessary for convergence of the adjoint-based inexact Newton (IN) type method in Algorithm 1, even when using an exact Hessian $H=\nabla_{y}^{2} \mathcal{L}(\bar{y}, \bar{\lambda})$. To support this statement, let us consider the following quadratic programming (QP) example from Potschka [39], based on a linear constraint $g(y)=\left[\begin{array}{ll}A_{1} & A_{2}\end{array}\right] y, n_{\mathrm{h}}=0$, and quadratic objective $f(y)=\frac{1}{2} y^{\top} H y$ in (1). The matrix $A_{1}$ is assumed invertible and close to identity, such that we can select the Jacobian approximation $M=\mathbb{1}_{n_{\mathrm{z}}} \approx A_{1}$. The problem data from [39] read as 


$$
\begin{aligned}
H & =\left[\begin{array}{cccc}
0.83 & 0.083 & 0.34 & -0.21 \\
0.083 & 0.4 & -0.34 & -0.4 \\
0.34 & -0.34 & 0.65 & 0.48 \\
-0.21 & -0.4 & 0.48 & 0.75
\end{array}\right], \\
A_{1} & =\left[\begin{array}{cc}
1.1 & 1.7 \\
0 & 0.52
\end{array}\right], A_{2}=\left[\begin{array}{cc}
-0.55 & -1.4 \\
-0.99 & -1.8
\end{array}\right] .
\end{aligned}
$$

For this specific QP instance, we may compute the linear contraction rate for the Newton-type method on the forward problem (3):

$$
\kappa_{\mathrm{F}}^{\star}=\rho\left(M^{-1} g_{z}-\mathbb{1}_{n_{\mathrm{z}}}\right)=\rho\left(A_{1}-\mathbb{1}_{n_{\mathrm{z}}}\right)=0.48<1 .
$$

In addition, let us consider the IN algorithm based on the solution of the linear system (8) for the same QP example using the exact Hessian $\tilde{H}=H$. We can then compute the corresponding contraction rate at the solution point:

$$
\kappa_{\mathrm{IN}}^{\star}=\rho\left(\tilde{J}_{\mathrm{IN}}^{-1} J-\mathbb{1}_{n_{\mathcal{F}}}\right) \approx 1.625>1,
$$

where $J=J(y, \lambda)$ denotes the exact Jacobian of the KKT system in (5). For this QP example (13), the Newton-type method on the forward problem locally converges with $\kappa_{\mathrm{F}}^{\star}=0.48<1$, while the corresponding IN algorithm is unstable with $\kappa_{\mathrm{IN}}^{\star} \approx 1.625>1$. In what follows, we present and study a novel Newton-type optimization algorithm based on iterated sensitivities, which circumvents this problem at a negligible additional computational cost. These observations are illustrated in Figure 1, which presents the Newton-type iterations for the different algorithms, starting from the same initial point and using the same Jacobian approximation. The figure includes the linear convergence for the Newton-type method (3) on the forward problem.

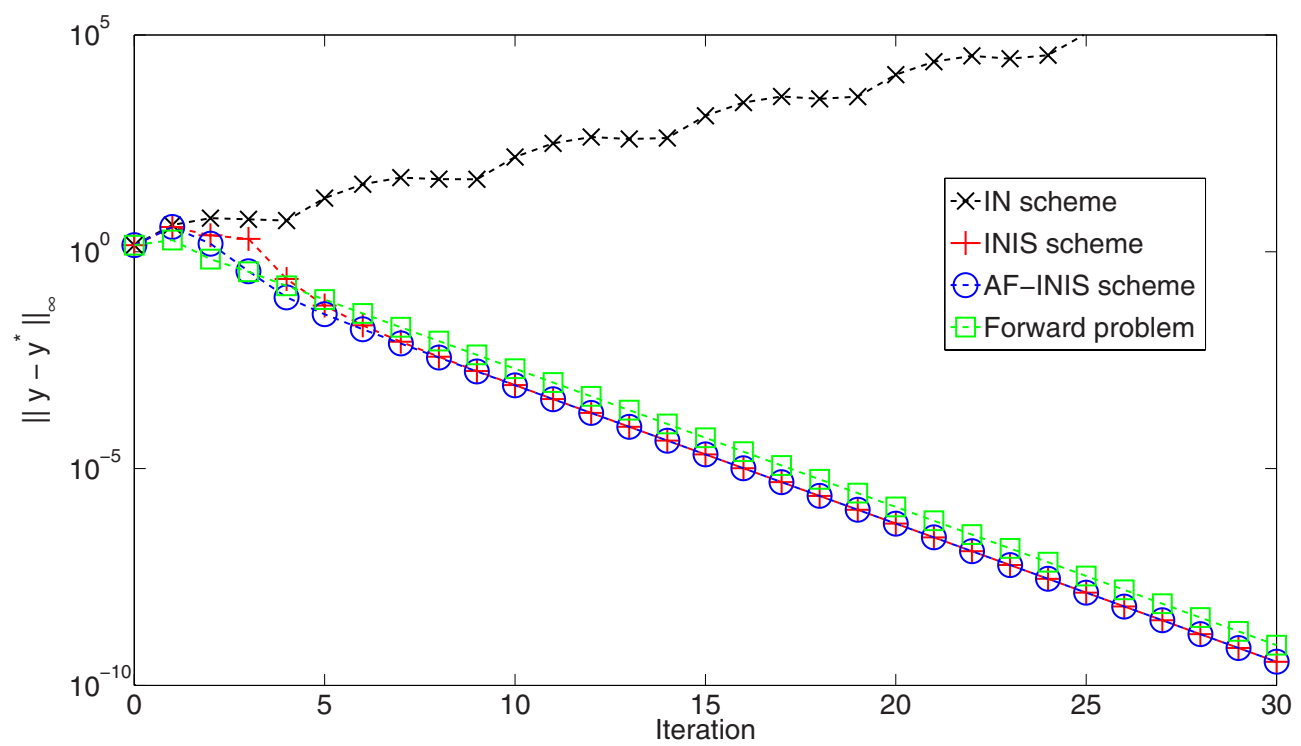

FIG. 1. Illustration of the divergence of the inexact Newton (IN) scheme and the convergence of the inexact Newton with iterated sensitivities (INIS) scheme for the QP in (13). In addition, the rate of convergence for INIS can be observed to be the same as for the forward problem. 
3. Inexact Newton with iterated sensitivities (INIS). Let us introduce an alternative inexact Newton-type optimization algorithm, labeled INIS in the following, based on the solution of an augmented KKT system defined as $^{1}$

$$
\mathcal{F}_{\text {INIS }}(y, \lambda, D)=\left[\begin{array}{c}
\nabla_{y} \mathcal{L}(y, \lambda) \\
c(y) \\
\operatorname{vec}\left(g_{z} D-g_{w}\right)
\end{array}\right]=0,
$$

where the additional variable $D \in \mathbb{R}^{n_{\mathrm{z}} \times n_{\mathrm{w}}}$ denotes the sensitivity matrix, implicitly defined by the equation $g_{z} D-g_{w}=0$. The number of variables in this augmented system is denoted by $n_{\mathrm{INIS}}=n_{\mathcal{F}}+n_{\mathrm{D}}$, where $n_{\mathcal{F}}=n_{\mathrm{y}}+n_{\mathrm{g}}+n_{\mathrm{h}}$ and $n_{\mathrm{D}}=n_{\mathrm{z}} n_{\mathrm{w}}$. The following proposition states the connection between the augmented system $\mathcal{F}_{\text {INIS }}(y, \lambda, D)=0$ and the original KKT system in (5).

Proposition 3.1. A regular point $\left(y^{\star}, \lambda^{\star}, D^{\star}\right)$ for the augmented system in (14), corresponds to a regular KKT point $\left(y^{\star}, \lambda^{\star}\right)$ for the NLP in (1).

Proof. This result follows directly from observing that the first two equations of the augmented system $\mathcal{F}_{\text {INIS }}(y, \lambda, D)=0$ correspond to the KKT conditions $\mathcal{F}(y, \lambda)=$ 0 in (5) for the original NLP problem in (1).

3.1. Implementation. We introduce the inexact Newton method with iterated sensitivities (INIS), to iteratively solve the augmented KKT system in (14) based on (15)

$$
\underbrace{\left[\begin{array}{ccc}
\tilde{H} & \left(\begin{array}{cc}
M^{\top} & h_{z}^{\top} \\
\bar{D}^{\top} M^{\top} & h_{w}^{\top}
\end{array}\right) & 0 \\
\left(\begin{array}{cc}
M & M \bar{D} \\
h_{z} & h_{w}
\end{array}\right) & 0 & 0 \\
0 & 0 & \mathbb{1}_{n_{\mathrm{w}}} \otimes M
\end{array}\right]}_{=: \tilde{J}_{\mathrm{INIS}}(\bar{y}, \bar{\lambda}, \bar{D})}\left[\begin{array}{c}
\Delta z \\
\Delta w \\
\Delta \mu \\
\Delta \nu \\
\operatorname{vec}(\Delta D)
\end{array}\right]=-\underbrace{\left[\begin{array}{c}
\nabla_{y} \mathcal{L}(\bar{y}, \bar{\lambda}) \\
c(\bar{y}) \\
\operatorname{vec}\left(g_{z} \bar{D}-g_{w}\right)
\end{array}\right]}_{=\mathcal{F}_{\mathrm{INIS}}(\bar{y}, \bar{\lambda}, \bar{D})},
$$

where $\otimes$ denotes the Kronecker product of matrices, and where we use the Jacobian approximation $M \approx g_{z}$ from the Newton-type method on the forward problem in (3). The resulting matrix $\tilde{J}_{\text {INIS }}(\bar{y}, \bar{\lambda}, \bar{D})$ forms an approximation for the exact Jacobian $J_{\text {INIS }}(\bar{y}, \bar{\lambda}, \bar{D}):=\frac{\partial \mathcal{F}_{\text {INIS }}}{\partial(y, \lambda, D)}(\bar{y}, \bar{\lambda}, \bar{D})$ of the augmented system. Similar to Remark 2.4, we assume that the Jacobian and Hessian approximations $M, \bar{D}$, and $\tilde{H}$ are such that the INIS matrix $\tilde{J}_{\text {INIS }}(\cdot)$ is continuously differentiable and invertible.

Algorithm 2 shows that the INIS scheme in (15) can be implemented efficiently using a condensing and expansion procedure and the computational cost can be made close to that of the standard inexact Newton method in Algorithm 1. More specifically, the INIS scheme requires the linear system solution $-M^{-1}\left(g_{z} \bar{D}-g_{w}\right)$, for which the right-hand side can be evaluated efficiently using AD techniques [28]. Similar to Remark 2.2, let us write the gradient correction in step 1 of Algorithm 2:

$$
\tilde{Z}^{\top} \nabla_{y} \mathcal{L}(\bar{y}, \bar{\lambda})=\tilde{Z}^{\top}\left(\nabla_{y} f(\bar{y})+\nabla_{y} h(\bar{y}) \bar{\nu}\right)-\left(g_{z} \bar{D}-g_{w}\right)^{\top} \bar{\mu},
$$

where $\tilde{Z}^{\top}:=\left[-\bar{D}^{\top}, \mathbb{1}_{n_{\mathrm{w}}}\right]$. Note that the evaluation of $g_{z} \bar{D}-g_{w}$ can be reused in steps 1 and 3 of Algorithm 2, which allows INIS to be computationally competitive with the standard IN scheme. This will also be illustrated by the numerical results for direct optimal control in section 5 .

\footnotetext{
${ }^{1}$ The operator $\operatorname{vec}(\cdot)$ denotes a vectorization of a matrix, i.e., this is a linear transformation that converts the matrix into a column vector.
} 
$\overline{\text { Algorithm 2. One iteration of an adjoint-based inexact Newton with iterated sen- }}$ sitivities (INIS) optimization method.

Input: Current values $\bar{y}=(\bar{z}, \bar{w}), \bar{\lambda}=(\bar{\mu}, \bar{\nu}), \bar{D}$ and approximations $M, \tilde{H}(\bar{y}, \bar{\lambda})$.

1: After eliminating the variables $\Delta z, \Delta \mu$ in (15), solve the resulting system:

$\left[\begin{array}{cc}\tilde{Z}^{\top} \tilde{H} \tilde{Z} & \tilde{Z}^{\top} h_{y}^{\top} \\ h_{y} \tilde{Z} & \mathbb{0}\end{array}\right]\left[\begin{array}{c}\Delta w \\ \Delta \nu\end{array}\right]=-\left[\begin{array}{c}\tilde{Z}^{\top} \nabla_{y} \mathcal{L}(\bar{y}, \bar{\lambda}) \\ h(\bar{y})\end{array}\right]-\left[\begin{array}{c}\tilde{Z}^{\top} \tilde{H} \\ h_{y}\end{array}\right]\left[\begin{array}{c}-M^{-1} g(\bar{y}) \\ \mathbb{0}\end{array}\right]$,

where $\tilde{Z}^{\top}:=\left[-\bar{D}^{\top}, \mathbb{1}_{n_{\mathrm{w}}}\right]$.

2: Based on $\Delta w$ and $\Delta \nu$, the corresponding values for $\Delta z$ and $\Delta \mu$ are found:

$\Delta z=-M^{-1} g(\bar{y})-\bar{D} \Delta w$ and

$\Delta \mu=-\left[\begin{array}{ll}M^{-\top} & 0\end{array}\right]\left(\nabla_{y} \mathcal{L}(\bar{y}, \bar{\lambda})+\tilde{H} \Delta y+h_{y}^{\top} \Delta \nu\right)$.

3: Independently, the sensitivity matrix is updated in each iteration:

$\Delta D=-M^{-1}\left(g_{z} \bar{D}-g_{w}\right)$.

Output: New values $\bar{y}^{+}=\bar{y}+\Delta y, \bar{\lambda}^{+}=\bar{\lambda}+\Delta \lambda$, and $\bar{D}^{+}=\bar{D}+\Delta D$.

3.2. Local contraction theorem. In what follows, we show that Algorithm 2 allows one to recover the connection between the contraction properties of the forward problem and the one of the Newton-type optimization algorithm. This observation makes the INIS-type optimization scheme depart fundamentally from the classical adjoint-based IN method. The local contraction of the forward problem will be shown to be necessary for the local convergence of the INIS algorithm, and can be sufficient under reasonable assumptions on the Hessian approximation $\tilde{H}$.

Let us formalize the local contraction rate $\kappa_{\text {INIS }}^{\star}=\rho\left(\tilde{J}_{\text {INIS }}^{-1} J_{\text {INIS }}-\mathbb{1}_{n_{\text {INIS }}}\right)$ for the INIS scheme (15), where the Jacobian of the augmented KKT system (14) reads

$$
J_{\text {INIS }}=\left[\begin{array}{ccc}
\nabla_{y}^{2} \mathcal{L} & c_{y}^{\top} & \mathbb{0} \\
c_{y} & \mathbb{0} & \mathbb{O} \\
s_{y} & \mathbb{0} & \mathbb{1}_{n_{\mathrm{w}}} \otimes g_{z}
\end{array}\right], \quad \text { where } s_{y}:=\frac{\partial}{\partial y} \operatorname{vec}\left(g_{z} D-g_{w}\right) .
$$

The following theorem specifies the eigenspectrum of the iteration matrix $\tilde{J}_{\text {INIS }}^{-1} J_{\text {INIS }}-$ $\mathbb{1}_{n_{\text {INIS }}}$ at the solution point $\left(y^{\star}, \lambda^{\star}, D^{\star}\right)$, using the notation $\sigma(P)$ to denote the spectrum, i.e., the set of eigenvalues for a matrix $P$.

THEOREM 3.2. For the augmented linear system (15) on the NLP in (1), the eigenspectrum of the INIS-type iteration matrix at the solution $\left(y^{\star}, \lambda^{\star}, D^{\star}\right)$ reads as

$$
\sigma\left(\tilde{J}_{\mathrm{INIS}}^{-1} J_{\mathrm{INIS}}-\mathbb{1}_{n_{\mathrm{INIS}}}\right)=\{0\} \cup \sigma\left(M^{-1} g_{z}-\mathbb{1}_{n_{\mathrm{z}}}\right) \cup \sigma\left(\tilde{H}_{Z}^{-1} H_{Z}-\mathbb{1}_{\tilde{n}_{\mathrm{Z}}}\right)
$$

where $\tilde{n}_{\mathrm{Z}}=n_{\mathrm{w}}-n_{\mathrm{h}}$ and $Z \in \mathbb{R}^{n_{\mathrm{y}} \times \tilde{n}_{\mathrm{Z}}}$ denotes a basis for the null space of the complete constraint Jacobian $c_{y}$, such that the reduced Hessians $H_{Z}:=Z^{\top} H Z \in \mathbb{R}^{\tilde{n}_{Z} \times \tilde{n}_{Z}}$ and $\tilde{H}_{Z}:=Z^{\top} \tilde{H} Z \in \mathbb{R}^{\tilde{n}_{\mathrm{Z}} \times \tilde{n}_{\mathrm{Z}}}$ are defined. Note that $H:=\nabla_{y}^{2} \mathcal{L}\left(y^{\star}, \lambda^{\star}\right)$ is the exact Hessian and $\tilde{H} \approx H$ is an approximation. More specifically, the iteration matrix has the $\tilde{n}_{\mathrm{Z}}$ eigenvalues of the matrix $\tilde{H}_{Z}^{-1} H_{Z}-\mathbb{1}_{\tilde{n}_{Z}}$ and the $n_{\mathrm{z}}$ eigenvalues of $M^{-1} g_{z}-\mathbb{1}_{n_{\mathrm{z}}}$ with an algebraic multiplicity of $\left(2+n_{\mathrm{w}}\right)$ and $\{0\}$ with algebraic multiplicity $\left(2 n_{\mathrm{h}}\right)$.

Proof. At the solution of the augmented KKT system for the NLP in (14), the sensitivity matrix corresponds to $D^{\star}=g_{z}^{-1} g_{w}$. We then introduce the following Jacobian matrix and its approximation: 


$$
g_{y}=\left[\begin{array}{ll}
g_{z} & g_{w}
\end{array}\right], \quad \tilde{g}_{y}=\left[\begin{array}{ll}
\mathbb{1}_{n_{\mathrm{z}}} & D^{\star}
\end{array}\right]=g_{z}^{-1} g_{y},
$$

such that the exact and inexact augmented Jacobian matrices read

$$
J_{\mathrm{INIS}}=\left[\begin{array}{cccc}
H & g_{y}^{\top} & h_{y}^{\top} & \mathbb{0} \\
g_{y} & \mathbb{0} & \mathbb{0} & \mathbb{0} \\
h_{y} & \mathbb{0} & \mathbb{0} & \mathbb{0} \\
s_{y} & \mathbb{0} & \mathbb{0} & \mathbb{1}_{n_{\mathrm{w}}} \otimes g_{z}
\end{array}\right], \quad \tilde{J}_{\mathrm{INIS}}=\left[\begin{array}{cccc}
\tilde{H} & \tilde{g}_{y}^{\top} M^{\top} & h_{y}^{\top} & \mathbb{0} \\
M \tilde{g}_{y} & \mathbb{0} & \mathbb{0} & \mathbb{0} \\
h_{y} & \mathbb{0} & \mathbb{0} & \mathbb{0} \\
\mathbb{0} & \mathbb{0} & \mathbb{0} & \mathbb{1}_{n_{\mathrm{w}}} \otimes M
\end{array}\right],
$$

at the solution point $\left(y^{\star}, \lambda^{\star}, D^{\star}\right)$. We observe that the eigenvalues $\gamma$ of the iteration matrix $\tilde{J}_{\text {INIS }}^{-1} J_{\text {INIS }}-\mathbb{1}_{n_{\text {INIS }}}$ are the zeros of

$$
\operatorname{det}\left(\tilde{J}_{\mathrm{INIS}}^{-1} J_{\mathrm{INIS}}-\mathbb{1}_{n_{\mathrm{INIS}}}-\gamma \mathbb{1}_{n_{\mathrm{INIS}}}\right)=\operatorname{det}\left(\tilde{J}_{\mathrm{INIS}}^{-1} J_{\mathrm{INIS}}-(\gamma+1) \mathbb{1}_{n_{\mathrm{INIS}}}\right)=0 .
$$

Since $\tilde{J}_{\text {INIS }}$ is invertible, the second equality holds if and only if

$$
\operatorname{det}\left(\tilde{J}_{\text {INIS }}\left(\tilde{J}_{\text {INIS }}^{-1} J_{\text {INIS }}-(\gamma+1) \mathbb{1}_{n_{\text {INIS }}}\right)\right)=\operatorname{det}\left(J_{\text {INIS }}-(\gamma+1) \tilde{J}_{\text {INIS }}\right)=0 .
$$

Using the notation in (19), we can rewrite the matrix $J_{\text {INIS }}-(\gamma+1) \tilde{J}_{\text {INIS }}$ as the following product of block matrices:

$$
\begin{aligned}
& J_{\text {INIS }}-(\gamma+1) \tilde{J}_{\text {INIS }} \\
& =\left[\begin{array}{cccc}
\mathbb{1}_{n_{\mathrm{y}}} & \mathbb{0} & \mathbb{0} & \mathbb{0} \\
\mathbb{0} & \tilde{M} & \mathbb{0} & \mathbb{0} \\
\mathbb{0} & \mathbb{0} & -\gamma \mathbb{1}_{n_{\mathrm{h}}} & \mathbb{0} \\
\mathbb{0} & \mathbb{0} & \mathbb{0} & \mathbb{1}_{n_{\mathrm{D}}}
\end{array}\right]\left[\begin{array}{cccc}
H-(\gamma+1) \tilde{H} & \tilde{g}_{y}^{\top} & h_{y}^{\top} & \mathbb{0} \\
\tilde{g}_{y} & \mathbb{0} & \mathbb{0} & \mathbb{0} \\
h_{y} & \mathbb{0} & \mathbb{0} & \mathbb{0} \\
s_{y} & \mathbb{0} & \mathbb{0} & \mathbb{1}_{n_{\mathrm{w}}} \otimes \tilde{M}
\end{array}\right] \\
& *\left[\begin{array}{cccc}
\mathbb{1}_{n_{\mathrm{y}}} & 0 & 0 & 0 \\
0 & \tilde{M} & 0 & 0 \\
0 & 0 & -\gamma \mathbb{1}_{n_{\mathrm{h}}} & 0 \\
0 & 0 & \mathbb{0} & \mathbb{1}_{n_{\mathrm{D}}}
\end{array}\right]^{\top}
\end{aligned}
$$

where the matrix $\tilde{M}=g_{z}-(\gamma+1) M$ is defined such that $\tilde{M} \tilde{g}_{y}=g_{y}-(\gamma+1) M \tilde{g}_{y}$. The determinant of the product of matrices in (20) can be rewritten as

$$
\begin{aligned}
& \operatorname{det}\left(J_{\mathrm{INIS}}-(\gamma+1) \tilde{J}_{\mathrm{INIS}}\right)=\operatorname{det}\left(\left[\begin{array}{cccc}
\mathbb{1}_{n_{\mathrm{y}}} & 0 & 0 & 0 \\
0 & \tilde{M} & 0 & 0 \\
0 & 0 & -\gamma \mathbb{1}_{n_{\mathrm{h}}} & 0 \\
0 & 0 & 0 & \mathbb{1}_{n_{\mathrm{D}}}
\end{array}\right]\right)^{2} \\
& * \operatorname{det}\left(\left[\begin{array}{cccc}
H-(\gamma+1) \tilde{H} & \tilde{g}_{y}^{\top} & h_{y}^{\top} & 0 \\
\tilde{g}_{y} & 0 & 0 & 0 \\
h_{y} & 0 & 0 & 0 \\
s_{y} & \mathbb{0} & 0 & \mathbb{1}_{n_{\mathrm{w}}} \otimes \tilde{M}
\end{array}\right]\right) \\
&=(-\gamma)^{2 n_{\mathrm{h}}} \operatorname{det}(\tilde{M})^{2+n_{\mathrm{w}}} \operatorname{det}\left(\left[\begin{array}{cccc}
H-(\gamma+1) \tilde{H} & \tilde{g}_{y}^{\top} & h_{y}^{\top} \\
\tilde{g}_{y} & \mathbb{0} & 0 \\
h_{y} & \mathbb{0} & \mathbb{0}
\end{array}\right]\right) .
\end{aligned}
$$

Note that the Jacobian approximation $M$ is invertible such that the determinant $\operatorname{det}(\tilde{M})$ is zero if and only if $\operatorname{det}\left(M^{-1} g_{z}-(\gamma+1) \mathbb{1}_{n_{z}}\right)=0$ holds. It follows that $\operatorname{det}\left(J_{\text {INIS }}-(\gamma+1) \tilde{J}_{\text {INIS }}\right)=0$ holds only for the values of $\gamma$ that fulfill 


$$
\begin{array}{rr}
\gamma=0 & \text { or } \\
\operatorname{det}\left(M^{-1} g_{z}-(\gamma+1) \mathbb{1}_{n_{z}}\right)=0 & \text { or } \\
\operatorname{det}\left(\left[\begin{array}{ccc}
H-(\gamma+1) \tilde{H} & \tilde{g}_{y}^{\top} & h_{y}^{\top} \\
\tilde{g}_{y} & 0 & 0 \\
h_{y} & \mathbb{0} & \mathbb{0}
\end{array}\right]\right)=0 .
\end{array}
$$

Note that (22b) is satisfied exactly for the eigenvalues $\gamma \in \sigma\left(M^{-1} g_{z}-\mathbb{1}_{n_{z}}\right)$ with an algebraic multiplicity $\left(n_{\mathrm{w}}+2\right)$ as can be observed directly in (21). It can be verified that the values for $\gamma$ satisfying (22c) are given by

$$
\operatorname{det}\left(Z^{\top}(H-(\gamma+1) \tilde{H}) Z\right)=\operatorname{det}\left(H_{Z}-(\gamma+1) \tilde{H}_{Z}\right)=0 \text {, }
$$

where $Z \in \mathbb{R}^{n_{y} \times \tilde{n}_{Z}}$ denotes a basis for the null space of the complete constraint Jacobian $c_{y}$. The last equality in (23) is satisfied only for the eigenvalues $\gamma \in$ $\sigma\left(\tilde{H}_{Z}^{-1} H_{Z}-\mathbb{1}_{\tilde{n}_{Z}}\right)$. Note that this, for example, corresponds to an additional eigenvalue $\gamma=0$ in the case of an exact Hessian matrix $\tilde{H}=H$.

Based on the latter results regarding the eigenspectrum of the iteration matrix, we now formally state the local contraction theorem for the proposed INIS method.

COROLlaRY 3.3 (Local INIS-type contraction). The local rate of convergence for the INIS-type optimization algorithm is defined by

$$
\kappa_{\text {INIS }}^{\star}=\rho\left(\tilde{J}_{\text {INIS }}^{-1} J_{\text {INIS }}-\mathbb{1}_{n_{\text {INIS }}}\right)=\max \left(\kappa_{\mathrm{F}}^{\star}, \rho\left(\tilde{H}_{Z}^{-1} H_{Z}-\mathbb{1}_{\tilde{n}_{Z}}\right)\right),
$$

where $\kappa_{\mathrm{F}}^{\star}=\rho\left(M^{-1} g_{z}-\mathbb{1}_{n_{z}}\right)$ is defined for the Newton-type method on the forward problem in (3). It follows that local contraction for the forward problem, i.e., $\kappa_{\mathrm{F}}^{\star}<1$, is necessary for local convergence of the INIS-type algorithm. Under the condition $\rho\left(\tilde{H}_{Z}^{-1} H_{Z}-\mathbb{1}_{\tilde{n}_{Z}}\right) \leq \kappa_{\mathrm{F}}^{\star}$ on the quality of the Hessian approximation, e.g., $\rho\left(\tilde{H}_{Z}^{-1} H_{Z}-\right.$ $\left.\mathbb{1}_{\tilde{n}_{Z}}\right)=0$ in the case of an exact Hessian, local contraction for the forward problem is sufficient since the asymptotic rate of convergence satisfies $\kappa_{\mathrm{INIS}}^{\star}=\kappa_{\mathrm{F}}^{\star}$.

3.3. Numerical results. Let us first revisit the motivating QP example from section 2.4, where the asymptotic contraction rate for the Newton-type method on the forward problem reads $\kappa_{\mathrm{F}}^{\star}=0.48<1$. In contrast, the solution was found to be asymptotically unstable since $\kappa_{\mathrm{IN}}^{\star} \approx 1.625>1$ for the IN method based on the same Jacobian approximation. Let us now consider the proposed INIS algorithm based on the solution of the linear system (15) for the same QP example using the exact Hessian $\tilde{H}=H$. We compute the corresponding contraction rate at the solution

$$
\kappa_{\text {INIS }}^{\star}=\rho\left(\tilde{J}_{\text {INIS }}^{-1} J_{\text {INIS }}-\mathbb{1}_{n_{\text {INIS }}}\right)=0.48<1,
$$

where $J_{\text {INIS }}$ denotes the exact Jacobian of the augmented KKT system in (17). Therefore, the INIS scheme indeed exhibits a linear local convergence with the same asymptotic rate as the forward problem, i.e., $\kappa_{\mathrm{INIS}}^{\star}=0.48=\kappa_{\mathrm{F}}^{\star}$. This result is consistent with Theorem 3.2 and is illustrated in Figure 1.

In addition, let us introduce a simple example of an NLP (1) based on the QP formulation above, where again $n_{\mathrm{h}}=0$. For this purpose, let us take a quadratic objective $f(y)=\frac{1}{2} y^{\top} H y+e^{\top} y$, where $H$ is defined in (13), the gradient vector $e=\left[\begin{array}{llll}0.1 & 0 & 0 & 0\end{array}\right]^{\top}$, and the nonlinear constraint function reads

$$
g(y)=\left[\begin{array}{ll}
A_{1} & A_{2}
\end{array}\right] y+0.1\left[\begin{array}{c}
y_{1}^{3} \\
y_{2} y_{4}
\end{array}\right],
$$




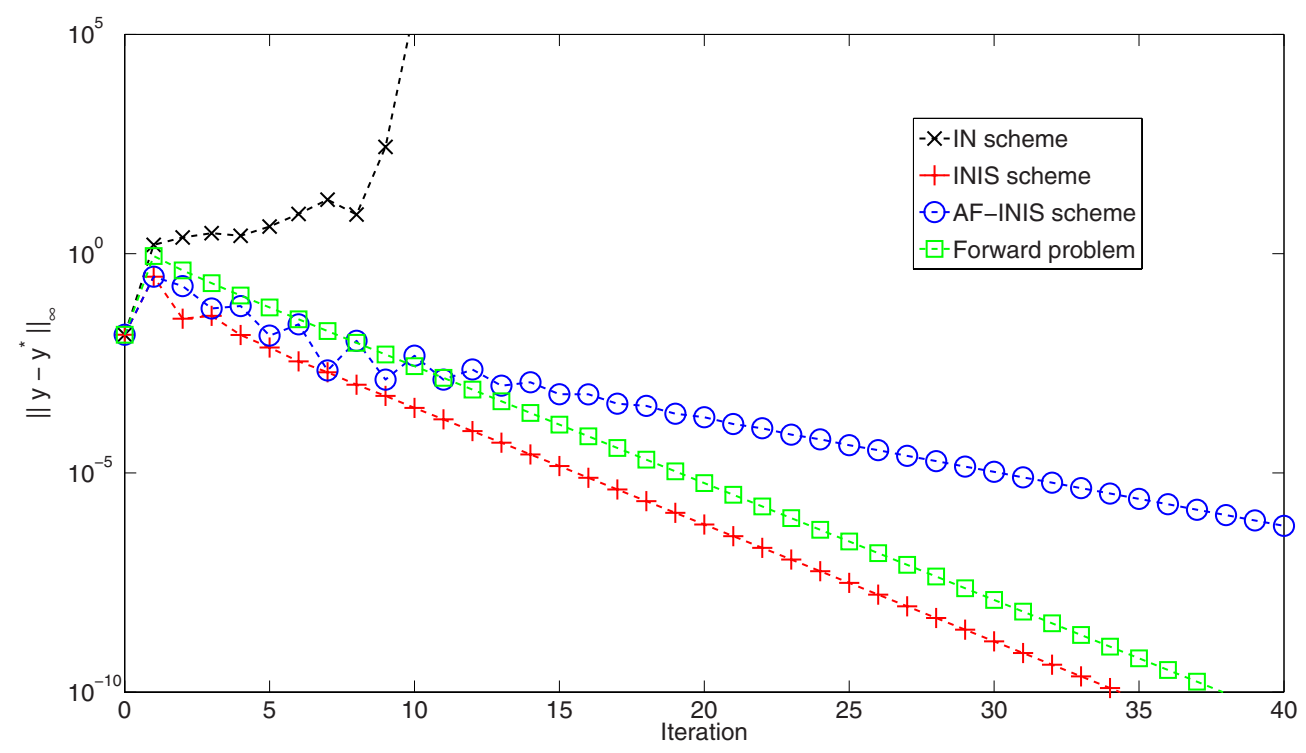

FIG. 2. Illustration of the divergence of the inexact Newton (IN) scheme and the convergence of the inexact Newton with iterated sensitivities (INIS) scheme for the NLP in (24). In addition, the rate of convergence for INIS can be observed to be the same as for the forward problem while the adjoint-free (AF-INIS) implementation has a different contraction rate for this NLP example.

where also the matrices $A_{1}$ and $A_{2}$ are adopted from (13). Figure 2 then illustrates the convergence results for the IN and INIS schemes from Algorithms 1 and 2 on this NLP example. It can be observed that the local contraction rate for INIS corresponds to that for the forward problem, while the standard IN implementation locally diverges for this particular example. More specifically, the asymptotic contraction rates at the NLP solution can be computed to be

$$
\kappa_{\mathrm{F}}^{\star}=\kappa_{\mathrm{INIS}}^{\star} \approx 0.541<1<1.441 \approx \kappa_{\mathrm{IN}}^{\star} .
$$

4. Adjoint-free INIS-type optimization. Algorithm 2 presented an INIStype Newton method to solve the augmented KKT system in (14), based on adjoint sensitivity propagation to evaluate the gradient of the Lagrangian $\nabla_{y} \mathcal{L}(y, \lambda)=$ $\nabla_{y} f(y)+\nabla_{y} g(y) \mu+\nabla_{y} h(y) \nu$. Unlike the standard IN method in Algorithm 1, for which adjoint sensitivity propagation is necessary for convergence as discussed in $[9,22]$, the proposed INIS algorithm allows for deploying an adjoint-free implementation as presented in this section. For this purpose, in order to motivate the use of such an adjoint-free INIS (AF-INIS) scheme, we assume the following.

- A multiplier-free Hessian approximation $\tilde{H}(y) \approx H(y, \lambda):=\nabla_{y}^{2} \mathcal{L}(y, \lambda)$ can be used for the NLP in (1), e.g., based on the generalized Gauss-Newton method $[8,37]$. It can be desirable to use a multiplier-free algorithm, which therefore does not require a good initialization of the multiplier values.

- The constraint function $g(\cdot)$ of the forward problem consists of a sequence of nontrivial operations, resulting in a Jacobian $g_{z}$ with a block banded structure. For example, in the case of direct optimal control [10], these constraints typically correspond to the numerical simulation of the system dynamics. Especially for implicit integration schemes, the computation of 
$\overline{\text { Algorithm 3. One iteration of an adjoint-free inexact Newton with iterated sensi- }}$ tivities (AF-INIS) optimization method.

Input: Current values $\bar{y}=(\bar{z}, \bar{w}), \bar{D}$ and approximations $M, \tilde{H}(\bar{y})$.

1: After eliminating the variables $\Delta z, \Delta \mu$ in (26), solve the resulting system:

$\left[\begin{array}{cc}\tilde{Z}^{\top} \tilde{H} \tilde{Z} & \tilde{Z}^{\top} h_{y}^{\top} \\ h_{y} \tilde{Z} & \mathbb{0}\end{array}\right]\left[\begin{array}{c}\Delta w \\ \bar{\nu}^{+}\end{array}\right]=-\left[\begin{array}{c}\tilde{Z}^{\top} \nabla_{y} f(\bar{y}) \\ h(\bar{y})\end{array}\right]-\left[\begin{array}{c}\tilde{Z}^{\top} \tilde{H} \\ h_{y}\end{array}\right]\left[\begin{array}{c}-M^{-1} g(\bar{y}) \\ \mathbb{0}\end{array}\right]$,

where $\tilde{Z}^{\top}:=\left[-\bar{D}^{\top}, \mathbb{1}_{n_{\mathrm{w}}}\right]$.

2: Based on $\Delta w$, the corresponding value for $\Delta z$ is found:

$\Delta z=-M^{-1} g(\bar{y})-\bar{D} \Delta w$.

3: Independently, the sensitivity matrix is updated in each iteration:

$\Delta D=-M^{-1}\left(g_{z} \bar{D}-g_{w}\right)$.

Output: New values $\bar{y}^{+}=\bar{y}+\Delta y$ and $\bar{D}^{+}=\bar{D}+\Delta D$.

adjoint derivatives typically results either in relatively high storage requirements of the forward variables or in an increased computational cost [45].

- Unlike the equations of the forward problem, the constraint function $h(\cdot)$ allows a relatively cheap evaluation of forward and adjoint derivatives.

The above assumptions are often satisfied for dynamic optimization problems, as discussed further in section 5. Even though any derivative in a Newton-type method could be evaluated either forward or backward, note that there is a clear motivation to avoid the use of adjoint differentiation specifically for the function $g(\cdot)$. However, we will show, including a counterexample, that such an adjoint-free INIS method generally cannot preserve the same asymptotic contraction rate for NLPs.

4.1. Implementation. Algorithm 3 presents the adjoint-free variant of the INIS optimization method from Algorithm 2. It corresponds to solving the following approximate variant of the augmented KKT system in (14):

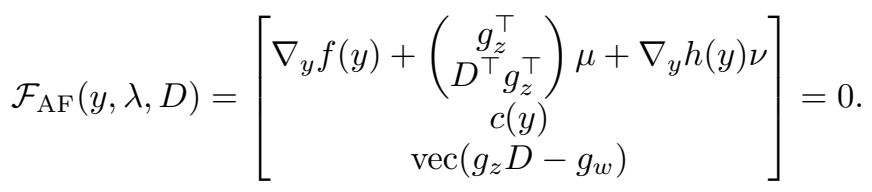

The following proposition formalizes the connection between this augmented system of equations and the original NLP in (1).

Proposition 4.1. A solution $\left(y^{\star}, \lambda^{\star}, D^{\star}\right)$ to the alternative augmented system in (25), corresponds to a regular KKT point $\left(y^{\star}, \lambda^{\star}\right)$ for the NLP in (1).

Proof. The third equation in both augmented KKT systems from (14) and (25) at the solution $\left(y^{\star}, \lambda^{\star}, D^{\star}\right)$ reads as $g_{z} D^{\star}-g_{w}=0$ such that $D^{\star}=g_{z}^{-1} g_{w}$ holds. The following equality therefore holds at the solution

$$
\nabla_{y} \mathcal{L}\left(y^{\star}, \lambda^{\star}\right)=\nabla_{y} f\left(y^{\star}\right)+\nabla_{y} c\left(y^{\star}\right) \lambda^{\star}=\nabla_{y} f\left(y^{\star}\right)+\left(\begin{array}{c}
g_{z}^{\top} \\
\left(g_{z} D^{\star}\right)^{\top}
\end{array}\right) \mu^{\star}+\nabla_{y} h\left(y^{\star}\right) \nu^{\star} .
$$

It follows that a solution of the adjoint-free augmented system (25) also forms a solution to the adjoint-based augmented system (14) and therefore is a regular KKT point for the NLP in (1) based on the result in Proposition 3.1. 
The adjoint-free inexact Newton method with iterated sensitivities (AF-INIS) then uses the same approximate Jacobian matrix $\tilde{J}_{\text {INIS }}(\bar{y}, \bar{\lambda}, \bar{D})$ from (15) to solve the augmented set of equations in (25). At each iteration, the corresponding linear system reads as

$$
\begin{aligned}
& \underbrace{\left[\begin{array}{ccc}
\tilde{H} & \left(\begin{array}{cc}
M^{\top} & h_{z}^{\top} \\
\bar{D}^{\top} M^{\top} & h_{w}^{\top}
\end{array}\right) & \mathbb{0} \\
\left(\begin{array}{cc}
M & M \bar{D} \\
h_{z} & h_{w}
\end{array}\right) & \mathbb{0} & \mathbb{0} \\
0 & \mathbb{1} & \mathbb{1}_{n_{\mathrm{w}}} \otimes M
\end{array}\right]}_{=\tilde{J}_{\mathrm{INIS}}(\bar{y}, \bar{\lambda}, \bar{D})}\left[\begin{array}{c}
\Delta z \\
\Delta w \\
\Delta \mu \\
\Delta \nu \\
\operatorname{vec}(\Delta D)
\end{array}\right] \\
& =-\underbrace{\left[\begin{array}{c}
\nabla_{y} f(\bar{y})+\left(\begin{array}{c}
g_{z}^{\top} \\
\bar{D}^{\top} g_{z}^{\top}
\end{array}\right) \bar{\mu}+\nabla_{y} h(\bar{y}) \bar{\nu} \\
c(\bar{y}) \\
\operatorname{vec}\left(g_{z} \bar{D}-g_{w}\right)
\end{array}\right]}_{=\mathcal{F}_{\mathrm{AF}}(\bar{y}, \bar{\lambda}, \bar{D})} .
\end{aligned}
$$

Using this augmented linear system, the steps $\Delta z, \Delta w$, and $\Delta D$ can be computed without evaluating adjoint derivatives for the function $g(\cdot)$ in Algorithm 3. The evaluation of these adjoint variables can be avoided because the following term vanishes when multiplying the first equation in the right-hand side of the latter system (26) by $\tilde{Z}^{\top}:=\left[-\bar{D}^{\top} \mathbb{1}_{n_{\mathrm{w}}}\right]$ :

$$
\left[\begin{array}{ll}
-\bar{D}^{\top} & \mathbb{1}_{n_{\mathrm{w}}}
\end{array}\right]\left(\begin{array}{c}
g_{z}^{\top} \\
\bar{D}^{\top} g_{z}^{\top}
\end{array}\right)=-\bar{D}^{\top} g_{z}^{\top}+\bar{D}^{\top} g_{z}^{\top}=\mathbb{0}
$$

resulting in an adjoint-free and multiplier-free computation in Algorithm 3. Note that the multipliers $\nu$ are also not needed, depending on how the linear system is solved in step 1 of the algorithm. Because we assumed the Hessian approximation $\tilde{H}(\bar{y})$ in this case to be independent of the current multiplier values, we can completely omit the computation of the update $\Delta \lambda$.

4.2. Local convergence results. Proposition 4.1 states that, if it converges, the adjoint-free implementation of the INIS method in Algorithm 3 converges to a local minimizer for the NLP in (1), and this is unlike standard adjoint-free inexact Newton methods as discussed in [9, 22]. Even though we will show that the result in Theorem 3.2 does not necessarily hold for the AF-INIS scheme applied to general NLPs, the following theorem extends this local contraction result for quadratic programming (QP) problems. Let us introduce the exact Jacobian of the adjoint-free augmented KKT system in (25):

$$
J_{\mathrm{AF}}(y, \lambda, D)=\left[\begin{array}{ccc}
f_{y y}+\tilde{g}_{y y}+h_{y y} & \left(\begin{array}{cc}
g_{z}^{\top} & h_{z}^{\top} \\
D^{\top} g_{z}^{\top} & h_{w}^{\top}
\end{array}\right) & \tilde{g}_{D} \\
\left(\begin{array}{cc}
g_{z} & g_{w} \\
h_{z} & h_{w}
\end{array}\right) & \mathbb{0} & \mathbb{0} \\
s_{y} & \mathbb{0} & \mathbb{1}_{n_{\mathrm{w}}} \otimes g_{z}
\end{array}\right],
$$

where the matrices $f_{y y}:=\nabla_{y}^{2} f(y), \tilde{g}_{y y}:=\frac{\partial}{\partial y}\left(\begin{array}{c}g_{z}^{\top} \mu \\ D^{\top} g_{z}^{\top} \mu\end{array}\right), h_{y y}:=\sum_{i=1}^{n_{\mathrm{h}}} \nabla_{y}^{2} h_{i}(y) \nu_{i}$, and

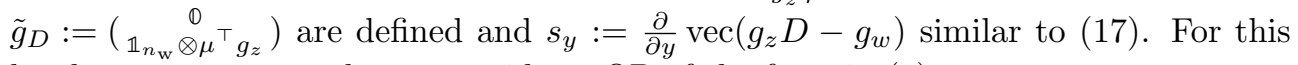
local convergence result, we consider a $\mathrm{QP}$ of the form in (1): 


$$
\begin{array}{ll}
\min _{z, w} & \frac{1}{2} y^{\top} H y+h^{\top} y \\
\text { s.t. } & A_{1} z+A_{2} w+a=0, \\
& B_{1} z+B_{2} w+b=0,
\end{array}
$$

where the matrix $A_{1}$ is assumed to be invertible and we have an invertible Jacobian approximation $M \approx A_{1}$ available.

ThEOREM 4.2. For the adjoint-free augmented linear system (26) corresponding to the QP in (28), the eigenspectrum of the AF-INIS iteration matrix reads

$$
\sigma\left(\tilde{J}_{\mathrm{INIS}}^{-1} J_{\mathrm{AF}}-\mathbb{1}_{n_{\mathrm{INIS}}}\right)=\{0\} \cup \sigma\left(M^{-1} A_{1}-\mathbb{1}_{n_{\mathrm{Z}}}\right) \cup \sigma\left(\tilde{H}_{Z}^{-1} H_{Z}-\mathbb{1}_{\tilde{n}_{\mathrm{Z}}}\right),
$$

at the solution $\left(y^{\star}, \lambda^{\star}, D^{\star}\right)$. The exact Jacobian $J_{\mathrm{AF}}(y, \lambda, D)$ is defined by (27) for which $s_{y}=\mathbb{O}, \tilde{g}_{y y}=\mathbb{O}, h_{y y}=\mathbb{O}$, and $f_{y y}=H$ in case of a $Q P$ formulation. Similar to Theorem 3.2, $\tilde{n}_{\mathrm{Z}}=n_{\mathrm{w}}-n_{\mathrm{h}}$ and $Z \in \mathbb{R}^{n_{\mathrm{y}} \times \tilde{n}_{\mathrm{Z}}}$ denotes a basis for the null space of the constraint Jacobian $\left[\begin{array}{l}A \\ B\end{array}\right]$, and $H_{Z}:=Z^{\top} H Z \in \mathbb{R}^{\tilde{n}_{Z} \times \tilde{n}_{Z}}$ and $\tilde{H}_{Z}:=Z^{\top} \tilde{H} Z \in \mathbb{R}^{\tilde{n}_{Z} \times \tilde{n}_{Z}}$.

The local rate of convergence for the adjoint-free INIS scheme on the QP formulation in (28) is defined by

$$
\kappa_{\mathrm{AF}}^{\star}=\rho\left(\tilde{J}_{\mathrm{INIS}}^{-1} J_{\mathrm{AF}}-\mathbb{1}_{n_{\mathrm{INIS}}}\right)=\max \left(\kappa_{\mathrm{F}}^{\star}, \rho\left(\tilde{H}_{Z}^{-1} H_{Z}-\mathbb{1}_{\tilde{n}_{\mathrm{Z}}}\right)\right) .
$$

Proof. At the solution of the adjoint-free augmented KKT system in (25) for the QP formulation in (28), we know that $D^{\star}=A_{1}^{-1} A_{2}$ and we use the notation $A=$ $\left[\begin{array}{ll}A_{1} & A_{2}\end{array}\right]$ and $\tilde{A}=A_{1}^{-1} A$. The eigenvalues $\gamma$ of the iteration matrix $\tilde{J}_{\mathrm{INIS}}^{-1} J_{\mathrm{AF}}-\mathbb{1}_{n_{\mathrm{INIS}}}$ are given by the expression $\operatorname{det}\left(J_{\mathrm{AF}}-(\gamma+1) \tilde{J}_{\mathrm{INIS}}\right)=0$, based on the exact and inexact adjoint-free augmented Jacobian matrices

$$
J_{\mathrm{AF}}=\left[\begin{array}{cccc}
H & A^{\top} & B^{\top} & \tilde{g}_{D} \\
A & \mathbb{0} & \mathbb{0} & 0 \\
B & \mathbb{0} & \mathbb{0} & \mathbb{0} \\
0 & \mathbb{0} & \mathbb{0} & \mathbb{1}_{n_{\mathrm{w}}} \otimes A_{1}
\end{array}\right], \tilde{J}_{\mathrm{INIS}}=\left[\begin{array}{cccc}
\tilde{H} & \tilde{A}^{\top} M^{\top} & B^{\top} & 0 \\
M \tilde{A} & 0 & 0 & 0 \\
B & 0 & 0 & 0 \\
0 & 0 & 0 & \mathbb{1}_{n_{\mathrm{w}}} \otimes M
\end{array}\right],
$$

where $\tilde{g}_{D}=\left(\underset{\mathbb{1}_{n_{\mathrm{w}}} \otimes \mu^{\star^{\top}}}{\stackrel{0}{*}} A_{1}\right)$ is defined at the solution point $\left(y^{\star}, \lambda^{\star}, D^{\star}\right)$. We can rewrite $J_{\mathrm{AF}}-(\gamma+1) \tilde{J}_{\mathrm{INIS}}$ as the following product of block matrices:

$$
\begin{aligned}
& J_{\mathrm{AF}}-(\gamma+1) \tilde{J}_{\mathrm{INIS}} \\
& =\left[\begin{array}{cccc}
\mathbb{1}_{n_{\mathrm{y}}} & \mathbb{0} & \mathbb{0} & \mathbb{0} \\
\mathbb{0} & \tilde{M} & \mathbb{0} & \mathbb{0} \\
\mathbb{0} & \mathbb{0} & -\gamma \mathbb{1}_{n_{\mathrm{h}}} & \mathbb{0} \\
\mathbb{0} & \mathbb{0} & \mathbb{0} & \mathbb{1}_{n_{\mathrm{D}}}
\end{array}\right]\left[\begin{array}{cccc}
H-(\gamma+1) \tilde{H} & \tilde{A}^{\top} & B^{\top} & \tilde{g}_{D} \\
\tilde{A} & \mathbb{0} & \mathbb{0} & \mathbb{0} \\
B & \mathbb{0} & \mathbb{0} & \mathbb{0} \\
\mathbb{0} & \mathbb{0} & \mathbb{0} & \mathbb{1}_{n_{\mathrm{w}}} \otimes \tilde{M}
\end{array}\right] \\
& *\left[\begin{array}{cccc}
\mathbb{1}_{n_{\mathrm{y}}} & 0 & 0 & 0 \\
0 & \tilde{M} & 0 & 0 \\
0 & 0 & -\gamma \mathbb{1}_{n_{\mathrm{h}}} & 0 \\
0 & 0 & 0 & \mathbb{1}_{n_{\mathrm{D}}}
\end{array}\right]^{\top}
\end{aligned}
$$

where $\tilde{M}=A_{1}-(\gamma+1) M$ is defined. The proof continues in the same way as for Theorem 3.2, since the determinant of this matrix can be written as

Copyright $@$ by SIAM. Unauthorized reproduction of this article is prohibited. 


$$
\begin{aligned}
& \operatorname{det}\left(J_{\mathrm{AF}}-(\gamma+1) \tilde{J}_{\mathrm{INIS}}\right) \\
& \quad=(-\gamma)^{2 n_{\mathrm{h}}} \operatorname{det}(\tilde{M})^{2+n_{\mathrm{w}}} \operatorname{det}\left(\left[\begin{array}{ccc}
H-(\gamma+1) \tilde{H} & \tilde{A}^{\top} & B^{\top} \\
\tilde{A} & 0 & 0 \\
B & 0 & 0
\end{array}\right]\right) .
\end{aligned}
$$

4.3. Remark on AF-INIS for NLPs. When applying the adjoint-free INIS scheme from Algorithm 3 to the NLP formulation in (1), the augmented system introduces off-diagonal blocks for the Jacobian matrix as defined in (27). Therefore, the local contraction result in Theorem 4.2 cannot be directly extended to the general NLP case, even though the practical convergence of AF-INIS can typically be expected to be similar for relatively mild nonlinearities in the problem formulation. Note that Figure 1 already illustrated the local convergence of the AF-INIS scheme on the QP in (13), for which the following holds:

$$
\kappa_{\mathrm{F}}^{\star}=\kappa_{\mathrm{INIS}}^{\star}=\kappa_{\mathrm{AF}}^{\star}=0.48<1<1.625 \approx \kappa_{\mathrm{IN}}^{\star} .
$$

Note that section 3.3 included a counterexample to the conjecture that Theorem 4.2 could hold for general NLPs. It can be observed from Figure 2 that the local convergence rate of AF-INIS is different from the adjoint based INIS scheme, i.e.,

$$
\kappa_{\mathrm{F}}^{\star}=\kappa_{\mathrm{INIS}}^{\star} \approx 0.541<\kappa_{\mathrm{AF}}^{\star} \approx 0.753<1<1.441 \approx \kappa_{\mathrm{IN}}^{\star},
$$

even though it still outperforms the standard inexact Newton (IN) method.

5. Applications and numerical results. This section motivates the practical applicability of the INIS-type optimization method, either with or without adjoint computation, respectively, in Algorithm 2 or 3. For this purpose, let us introduce simultaneous direct optimal control methods for the popular class of dynamic optimization problems which typically have the form in (1), where the functions $f(\cdot), g(\cdot)$, and $h(\cdot)$ are twice continuously differentiable and the Jacobian matrix $g_{z}$ is invertible. Similar to before, this discussion omits the presence of inequality constraints even though the above results on local Newton-type convergence can be extended. This will be illustrated based on numerical results for the chain mass example [49].

5.1. Direct optimal control. In direct optimal control [10], one applies a firstdiscretize-then-optimize type of approach where one first discretizes the continuous time optimal control problem (OCP) such that one can subsequently solve an NLP of the form in (1). In case of direct collocation [5], such a discrete-time OCP problem can, for example, read as

$$
\begin{array}{rll}
\min _{X, U, K} & \sum_{i=0}^{N-1} l_{i}\left(x_{i}, u_{i}\right)+l_{N}\left(x_{N}\right) & \\
\text { s.t. } & 0=c_{i}\left(x_{i}, u_{i}, K_{i}\right), & i=0, \ldots, N-1, \\
& 0=x_{0}-\hat{x}_{0}, & \\
& 0=x_{i}+B_{i} K_{i}-x_{i+1}, \quad i=0, \ldots, N-1,
\end{array}
$$

with differential states $x_{i} \in \mathbb{R}^{n_{x}}$, control inputs $u_{i} \in \mathbb{R}^{n_{u}}$, and collocation variables $K_{i} \in \mathbb{R}^{q N_{\mathrm{s}} n_{x}}$, in which $q$ is the number of collocation nodes and $N_{\mathrm{s}}$ the amount of integration steps. In addition, the state $X=\left[x_{0}^{\top}, \ldots, x_{N}^{\top}\right]^{\top}$ and control trajectory 
$U=\left[u_{0}^{\top}, \ldots, u_{N-1}^{\top}\right]^{\top}$ and the trajectory of collocation variables $K=\left[K_{0}^{\top}, \ldots, K_{N-1}^{\top}\right]^{\top}$ are defined. The function $l_{i}(\cdot)$ denotes the stage cost and $c_{i}: \mathbb{R}^{n_{x}} \times \mathbb{R}^{n_{u}} \times \mathbb{R}^{n_{K}} \rightarrow \mathbb{R}^{n_{K}}$ defines the collocation polynomial on each interval $i=0, \ldots, N-1$, where $N$ denotes the number of intervals in the control horizon.

When comparing this OCP to the general NLP formulation in (1), similar to the detailed discussion in [44], one can relate the variables $z=\left[K_{0}^{\top}, \ldots, K_{N-1}^{\top}\right]^{\top}$ and $w=\left[x_{0}^{\top}, u_{0}^{\top}, \ldots, u_{N-1}^{\top}, x_{N}^{\top}\right]^{\top}$. Given the state and control values in $w$, the nonlinear collocation equations (33b) form the function $g(\cdot)$ that defines all variables in $z$ as required for the problem formulation in (1). The additional equality constraints from (1c) then correspond to the initial value constraint in (33c) and the continuity constraints in (33d). Based on Remark 2.5, note that the Newton-type local convergence results in this article still hold for inequality constrained optimization problems under certain regularity conditions in a neighborhood of the local minimizer. This will also be illustrated numerically in subsection 5.3. The Newton-type optimization algorithms in this article can rely on an efficient approximation of the invertible Jacobian $M_{i} \approx \frac{\partial c_{i}}{\partial K_{i}}$ as discussed in $[3,12,13,26,42]$ for collocation methods. These collocation variables could be numerically eliminated in each iteration, based on the constraints in (33b), resulting in a multiple shooting type method as discussed in [43, 44]. It is important to note that the sensitivity matrix variable in INIS-type optimization has a block-diagonal structure here because of the stage-wise definition of the collocation equations in (33b), i.e., $D_{i} \in \mathbb{R}^{n_{K} \times\left(n_{x}+n_{u}\right)}$ can be defined for $i=0, \ldots, N-1$. In addition, the conditions in section 4 are satisfied, for example, in the case of a (nonlinear) least squares type objective in (33a), for which a Gauss-Newton Hessian approximation can be used. The constraints in (33c) and (33d) are linear in this OCP formulation, while the nonlinear collocation equations in (33b) can correspond to a sequence of integration steps for which adjoint differentiation could be avoided in the AF-INIS optimization algorithm.

Similar to the formulation in [44], we can write the collocation equations in (33b) for one interval $i=0, \ldots, N-1$ :

$$
c_{i}\left(x_{i}, u_{i}, K_{i}\right)=\left[\begin{array}{c}
c_{i, 1}\left(x_{i, 0}, u_{i}, K_{i, 1}\right) \\
\vdots \\
c_{i, N_{\mathrm{s}}}\left(x_{i, N_{\mathrm{s}}-1}, u_{i}, K_{i, N_{\mathrm{s}}}\right)
\end{array}\right]=0
$$

for $N_{\mathrm{s}}$ integration steps of a $q$-stage collocation method. Note that $x_{i, j} \in \mathbb{R}^{n_{x}}$ for $j=1, \ldots, N_{\mathrm{s}}$ denote the intermediate state values, $x_{i, 0}=x_{i}$, and $K_{i, j} \in \mathbb{R}^{q n_{x}}$ is defined such that $K_{i}=\left[K_{i, 1}^{\top}, \ldots, K_{i, N_{s}}^{\top}\right]^{\top}$. This sequential simulation structure in (34) results in a constraint Jacobian that is block banded, as well as its approximation $M_{i} \approx \frac{\partial c_{i}}{\partial K_{i}}$. As discussed in detail in [44], this particular structure can be exploited by performing a forward and a backward propagation sweep, respectively, for the condensing and the expansion step of the adjoint-based schemes in Algorithms 1 and 2. In the case of an adjoint-free implementation, based on Algorithm 3, this procedure instead reduces to a forward propagation sweep.

5.2. ACADO code generation tool. An open-source implementation of the INIS-type optimization algorithm for the direct collocation based OCP formulation in (33) is part of the ACADO toolkit [32]. Presented as lifted collocation integrators in [44], the methods have more specifically been implemented as part of the ACADO code generation tool. This package can be used to obtain real-time feasible code for dynamic optimization on embedded control hardware. In particular, it pursues the 
TABLE 1

Average timing results per Gauss-Newton based SQP iteration on the chain mass problem using direct collocation $\left(N_{\mathrm{s}}=3, q=4\right)$, including different numbers of masses $n_{\mathrm{m}}$ and states $n_{x}$.

\begin{tabular}{ll|c|c|c|c}
\hline$n_{\mathrm{m}}$ & $n_{x}$ & Gauss-Newton & IN & INIS & AF-INIS \\
\hline 3 & 12 & $5.33 \mathrm{~ms}$ & $2.40 \mathrm{~ms}$ & $2.19 \mathrm{~ms}$ & $1.95 \mathrm{~ms}$ \\
4 & 18 & $14.79 \mathrm{~ms}$ & $5.43 \mathrm{~ms}$ & $4.76 \mathrm{~ms}$ & $4.29 \mathrm{~ms}$ \\
5 & 24 & $34.04 \mathrm{~ms}$ & $10.71 \mathrm{~ms}$ & $9.39 \mathrm{~ms}$ & $7.96 \mathrm{~ms}$ \\
6 & 30 & $62.08 \mathrm{~ms}$ & $18.73 \mathrm{~ms}$ & $14.88 \mathrm{~ms}$ & $12.71 \mathrm{~ms}$ \\
7 & 36 & $106.57 \mathrm{~ms}$ & $36.09 \mathrm{~ms}$ & $21.93 \mathrm{~ms}$ & $20.06 \mathrm{~ms}$ \\
\hline
\end{tabular}

export of efficient C-code based on the real-time iteration (RTI) scheme for nonlinear MPC (NMPC) [21, 33]. This online algorithm is based on sequential quadratic programming (SQP) to solve the nonlinear optimization problem within direct multiple shooting [10]. Regarding the INIS-type implementation following Algorithms 2 and 3, tailored Jacobian approximations are used for collocation methods, based on either simplified or single Newton-type iterations as presented in [42]. As discussed earlier in section 4.1, a multiplier-free Hessian approximation such as in the Generalized GaussNewton method [8] is used for the adjoint-free variant (AF-INIS). The standard INIS algorithm can rely on any approximation technique, including an exact Hessian based approach [37]. Similar to the implementation described in Algorithms 2 and 3, condensing and expansion techniques are used to obtain multiple shooting structured subproblems in each iteration of the SQP algorithm [44]. Tailored convex solvers such as qPOASES [23], qPDUNES [24], and HPMPC [25] can be used to solve these subproblems, especially in the presence of inequality constraints.

5.3. Numerical results: Chain of masses. We consider the chain mass optimal control problem from [44, 49]. The objective is to return a chain of $n_{\mathrm{m}}$ masses connected with springs to its steady state, starting from a perturbed initial configuration. The mass at one end is fixed, while the control input $u \in \mathbb{R}^{3}$ to the system is the direct force applied to the mass at the other end of the chain. The state of each free mass $x^{j}:=\left[{p^{j^{\top}}}_{v^{j^{\top}}}\right]^{\top} \in \mathbb{R}^{6}$ for $j=1, \ldots, n_{\mathrm{m}}-1$ consists of its position and velocity, such that the dynamic system can be described by the concatenated state vector $x(t) \in \mathbb{R}^{6\left(n_{\mathrm{m}}-1\right)}$. More details on the resulting model equations can be found in [49]. The OCP problem formulation is adopted from [44]. In addition to the constraints in (33), this OCP includes simple bounds on the control inputs and the path constraint that the chain should not hit a wall placed close to the equilibrium state. The ACADO code generation tool is used to generate an SQP type algorithm to solve the resulting inequality constrained optimization problem. Since the stage cost in the objective (33a) represents minimizing the control effort in the least squares sense, a Gauss-Newton based Hessian approximation will be used in this numerical case study. In addition, each SQP subproblem is solved using the parametric activeset solver qpDASES [23] in combination with a condensing technique to numerically eliminate the state variables [10].

Table 1 presents average timing results per Gauss-Newton based SQP iteration of the automatic generated solver using the ACADO toolkit, for different numbers of masses $n_{\mathrm{m}} \cdot{ }^{2}$ Note that the IN, INIS, and AF-INIS schemes correspond to the proposed implementations in Algorithms 1, 2, and 3, based on the lifted collocation

\footnotetext{
${ }^{2}$ All numerical simulations are carried out on a standard computer, equipped with an Intel i73720QM processor, using a 64-bit version of Ubuntu 14.04 and the $\mathrm{g}++$ compiler version 4.8.4.
} 


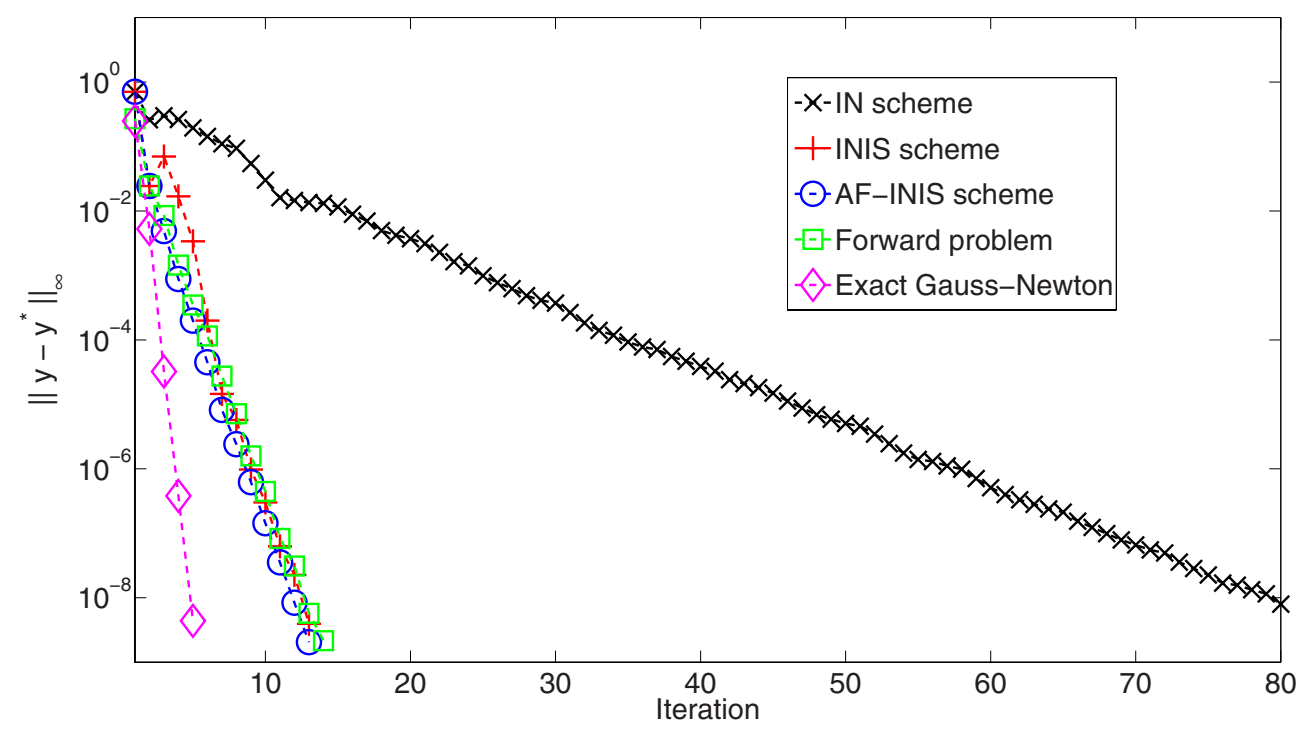

FIG. 3. Convergence results of the Gauss-Newton based SQP method with different inexact Newton-type techniques for the chain mass optimal control problem using $n_{\mathrm{m}}=4$ masses.

integrators as presented in [44]. On the other hand, the exact Gauss-Newton method in this case is based on a direct solution of the QP subproblem, corresponding to the linearized KKT conditions (6) including a Gauss-Newton Hessian approximation. The table shows that the use of inexact Jacobian approximations, tailored for collocation methods [42], can considerably reduce the computational effort over an exact implementation. More specifically, the single Newton implementation from [27] has been used for the four-stage Gauss collocation scheme $(q=4)$. A speedup of about factor 5 can be observed for the INIS-type scheme on this particular example. Figure 3 illustrates the convergence results for the SQP method, based on these different Newton-type optimization techniques. The figure shows a simulation result for which the inexact Newton (IN) scheme still results in local convergence, even though the contraction rate can be observed to be considerably slower than both of the variants of the proposed INIS algorithm.

Note that the Gauss-Newton based Hessian approximation does not depend on the multipliers for the equality constraints, but the convergence of both the adjointbased IN and INIS scheme in Algorithms 1 and 2 does depend on the initialization of these Lagrange multipliers unlike the adjoint-free (AF-INIS) variant. For simplicity, these multipliers have been initialized using zero values to obtain the numerical results in this case study. This difference in convergence behavior can also be observed in Figure 3. Even though the convergence for both INIS-type variants is close to that for the Newton-type method on the forward problem of this example, the contraction result in Theorem 3.2 cannot generally be extended to the AF-INIS algorithm for nonlinear optimization as discussed in section 4.3. The results for the exact Gauss-Newton method have been included mainly as a reference. It namely induces a relatively high computational cost as illustrated by Table 1, especially in cases where only rather low accuracy results are sufficient.

6. Conclusions. This article presented a novel family of optimization algorithms, based on inexact Newton-type iterations with iterated sensitivities (INIS). 
Unlike standard inexact Newton methods, this technique is shown to preserve the local contraction properties of the forward problem, based on a specific Jacobian approximation for the corresponding equality constraints. More specifically, local convergence for the Newton-type method on the forward problem is shown to be necessary and, under mild conditions, even sufficient for the asymptotic contraction of the corresponding INIS-type optimization algorithm. The article presents how this INIS algorithm can be implemented efficiently, resulting in a computational cost close to that of the standard inexact Newton implementation. In addition, an adjointfree (AF-INIS) variant is proposed and its local convergence properties are studied. This alternative approach can be preferable whenever the algorithm can be carried out independently of the current values for the multipliers corresponding to the equality constraints. Finally, an open-source implementation of these INIS-type techniques for simultaneous direct optimal control has been presented as part of the ACADO toolkit. Theoretical results are illustrated using toy examples of optimization problems, in addition to the benchmark case study of the optimal control for a chain of masses.

\section{REFERENCES}

[1] J. Albersmeyer and M. Diehl, The lifted Newton method and its application in optimization, SIAM J. Optim., 20 (2010), pp. 1655-1684.

[2] J. BetTs, Practical Methods for Optimal Control and Estimation Using Nonlinear Programming, 2nd ed., SIAM, Philadelphia, 2010.

[3] T. A. BICKART, An efficient solution process for implicit Runge-Kutta methods, SIAM J. Numer. Anal., 14 (1977), pp. 1022-1027.

[4] L. Biegler, O. Ghattas, M. Heinkenschloss, and B. van Bloemen WaAnders, eds., LargeScale PDE-Constrained Optimization, Lect. Notes Comput. Sci. Eng. 30, Springer-Verlag, Berlin, 2003.

[5] L. T. Biegler, Nonlinear Programming, MOS-SIAM Ser. Optim., SIAM, Philadelphia, 2010.

[6] H. Bock, Randwertproblemmethoden zur Parameteridentifizierung in Systemen nichtlinearer Differentialgleichungen, Bonner Math. Schrift. 183, Universität Bonn, Bonn, 1987.

[7] H. Bock, W. Egartner, W. Kappis, And V. Schulz, Practical shape optimization for turbine and compressor blades by the use of PRSQP methods, Optim. Eng., 3 (2002), pp. 395-414.

[8] H. G. Bock, Recent Advances in Parameter Identification Techniques for ODE, in Numerical Treatment of Inverse Problems in Differential and Integral Equations, Birkhäuser, Basel, 1983, pp. 95-121.

[9] H. G. Bock, M. Diehl, E. A. Kostina, And J. P. Schlöder, Constrained optimal feedback control of systems governed by large differential algebraic equations, in Real-Time and Online PDE-Constrained Optimization, SIAM, Philadelphia, 2007, pp. 3-22.

[10] H. G. Bock AND K. J. PLITT, A multiple shooting algorithm for direct solution of optimal control problems, in Proceedings of the IFAC World Congress, Pergamon Press, Oxford, 1984, pp. 242-247.

[11] P. T. Boggs and J. W. Tolle, Sequential quadratic programming, Acta Num., 4 (1995), pp. 1-51.

[12] J. Butcher, On the implementation of implicit Runge-Kutta methods, BIT Numer. Math., 16 (1976), pp. 237-240.

[13] G. Cooper and R. Vignesvaran, Some schemes for the implementation of implicit RungeKutta methods, J. Comput. Appl. Math., 45 (1993), pp. 213-225.

[14] F. E. Curtis, T. C. Johnson, D. P. Robinson, and A. WÄChter, An inexact sequential quadratic optimization algorithm for nonlinear optimization, SIAM J. Optim., 24 (2014), pp. 1041-1074.

[15] F. E. Curtis, J. Nocedal, And A. Wächter, A matrix-free algorithm for equality constrained optimization problems with rank-deficient Jacobians, SIAM J. Optim., 20 (2009), pp. 1224-1249.

[16] R. Dembo, S. Eisenstat, and T. Steinaug, Inexact Newton methods, SiAM J. Numer. Anal., 19 (1982), pp. 400-408.

[17] J. E. Dennis, On Newton-like methods, Numer. Math., 11 (1968), pp. 324-330.

[18] J. E. Dennis and J. J. MoRé, Quasi-Newton methods, motivation and theory, SIAM Rev., 19 (1977), pp. 46-89. 
[19] P. Deuflhard, Newton Methods for Nonlinear Problems: Affine Invariance and Adaptive Algorithms, Springer Ser. Comput. Math. 35, Springer-Verlag, Berlin, 2011.

[20] M. DieHL, Lecture Notes on Numerical Optimization, http://cdn.syscop.de/publications/ Diehl2016.pdf (2016).

[21] M. Diehl, H. G. Bock, J. Schlöder, R. Findeisen, Z. Nagy, and F. Allgöwer, Real-time optimization and nonlinear model predictive control of processes governed by differentialalgebraic equations, J. Process Control, 12 (2002), pp. 577-585.

[22] M. Diehl, A. Walther, H. G. Bock, AND E. Kostina, An adjoint-based SQP algorithm with quasi-Newton Jacobian updates for inequality constrained optimization, Optim. Methods Softw., 25 (2010), pp. 531-552.

[23] H. J. Ferreau, C. Kirches, A. Potschka, H. G. Bock, and M. Diehl, qpOASES: A parametric active-set algorithm for quadratic programming, Math. Program. Comput., 6 (2014), pp. 327-363.

[24] J. V. Frasch, S. SAger, And M. Diehl, A parallel quadratic programming method for dynamic optimization problems, Math. Program. Comput., 7 (2015), pp. 289-329.

[25] G. Frison, H. B. Sorensen, B. Dammann, and J. B. Jørgensen, High-performance smallscale solvers for linear model predictive control, in Proceedings of the European Control Conference (ECC), June 2014, European Control Association, pp. 128-133.

[26] S. GonzÁlez-Pinto, J. I. Montijano, And L. RÁndez, Iterative schemes for three-stage implicit Runge-Kutta methods, Appl. Numer. Math., 17 (1995), pp. 363-382.

[27] S. González-Pinto, S. PÉrez-Rodríguez, and J. I. MontiJano, Implementation of highorder implicit Runge-Kutta methods, Comput. Math. Appl., 41 (2001), pp. 1009-1024.

[28] A. GRIEWANK, Evaluating derivatives, principles and techniques of algorithmic differentiation, Front. Appl. Math. 19, SIAM, Philadelphia, 2000.

[29] A. Griewank and A. Walther, On constrained optimization by adjoint based quasi-Newton methods, Optim. Methods Softw., 17 (2002), pp. 869-889.

[30] M. Heinkenschloss And L. Vicente, Analysis of inexact trust-region SQP algorithms, SIAM J. Optim., 12 (2001), pp. 283-302.

[31] B. Houska AND M. DieHL, A quadratically convergent inexact SQP method for optimal control of differential algebraic equations, Optim. Control Appl. Methods, 34 (2013), pp. 396-414.

[32] B. Houska, H. J. Ferreau, And M. Diehl, ACADO toolkit - An open source framework for automatic control and dynamic optimization, Optimal Control Appl. Methods, 32 (2011), pp. 298-312.

[33] B. Houska, H. J. Ferreau, And M. Diehl, An auto-generated real-time iteration algorithm for nonlinear MPC in the microsecond range, Automatica, 47 (2011), pp. 2279-2285.

[34] H. JaEger And E. SAChS, Global convergence of inexact reduced SQP methods, Optim. Methods Softw., 7 (1997), pp. 83-110.

[35] T. C. Johnson, C. Kirches, And A. Wächter, An active-set method for quadratic programming based on sequential hot-starts, SIAM J. Optim., 25 (2015), pp. 967-994.

[36] F. LeIBfritz AND E. W. SAChs, Inexact SQP interior point methods and large scale optimal control problems, SIAM J. Control Optim., 38 (2006), pp. 272-293.

[37] J. Nocedal and S. J. Wright, Numerical Optimization, 2nd ed., Springer Ser. Oper. Res. Financ. Eng., Springer-Verlag, Berlin, 2006.

[38] A. Potschka, Handling Path Constraints in a Direct Multiple Shooting Method for Optimal Control Problems, Diplomarbeit, University of Heidelberg, Heidelberg, 2006.

[39] A. Potschka, A Direct Method for the Numerical Solution of Optimization Problems with Time-Periodic PDE Constraints, Ph.D. thesis, University of Heidelberg, Heidelberg, 2011.

[40] A. Potschka, H. Bock, S. Engell, A. Küpper, And J. Schlöder, Optimization of Periodic Adsorption Processes: The Newton-Picard Inexact SQP Method, Preprint of DFG Priority Programme 1253: Optimization with Partial Differential Equations, SPP1253-01-01, 2008.

[41] R. Quirynen, Numerical Simulation Methods for Embedded Optimization, Ph.D. thesis, KU Leuven and University of Freiburg, 2017.

[42] R. Quirynen, S. Gros, And M. Dienl, Inexact Newton based lifted implicit integrators for fast nonlinear MPC, in Proceedings of the IFAC Conference on Nonlinear Model Predictive Control (NMPC), 2015, International Federation of Automatic Control, pp. 32-38.

[43] R. Quirynen, S. Gros, And M. Diehl, Lifted implicit integrators for direct optimal control, in Proceedings of the IEEE Conference on Decision and Control (CDC), IEEE, Piscataway, NJ, 2015, pp. 3212-3217.

[44] R. Quirynen, S. Gros, B. Houska, And M. Diehl, Lifted collocation integrators for direct optimal control in ACADO toolkit, Math. Program. Comput., 9 (2017), pp. 527-571.

[45] R. Quirynen, B. Houska, And M. Diehl, Efficient symmetric Hessian propagation for direct optimal control, J. Process Control, 50 (2017), pp. 19-28.

Copyright $@$ by SIAM. Unauthorized reproduction of this article is prohibited. 
[46] S. Robinson, Perturbed Kuhn-Tucker points and rates of convergence for a class of nonlinear programming algorithms, Math. Program., 7 (1974), pp. 1-16.

[47] A. Walther And L. Biegler, Numerical experiments with an inexact Jacobian trust-region algorithm, Comput. Optim. Appl., 48 (2011), pp. 255-271.

[48] A. Walther, S. R. R. Vetukuri, and L. T. Biegler, A first-order convergence analysis of trust-region methods with inexact Jacobians and inequality constraints, Optim. Methods Softw., 27 (2012), pp. 373-389.

[49] L. Wirsching, H. G. Bock, And M. Diehl, Fast NMPC of a chain of masses connected by springs, in Proceedings of the IEEE International Conference on Control Applications, Munich, IEEE, Piscataway, NJ, 2006, pp. 591-596.

Copyright (c) by SIAM. Unauthorized reproduction of this article is prohibited. 